\title{
Complex Solid and Cystic Breast Cancer: A Series of Six Case Reports
}

\author{
Nishi Mehta ${ }^{1 *}$, Lee Rousslang ${ }^{1}$, Mohammad Shokouh-Amiri ${ }^{2}$, Elizabeth L Wiley ${ }^{2}$, Lauren Green ${ }^{1}$ \\ 1. Department of Radiology, University of Illinois Hospital and Health Sciences System, Chicago, USA \\ 2. Department of Pathology, University of Illinois Hospital and Health Sciences System, Chicago, USA \\ * Correspondence: Nishi Mehta, M.D., University of Illinois Hospital and Health Sciences System, 1740 W Taylor Street, Chicago, IL \\ 60612, USA \\ (\mehta511@gmail.com)
}

Radiology Case. 2020 Feb; 14(2):21-44 :: $\quad$ DOI: $10.3941 /$ jrcr.v14i2.3712

\begin{abstract}
A cyst in the breast containing a thick wall, internal septations, or a solid intracystic component is defined as a complex solid and cystic breast mass. These lesions carry a malignant potential between $23-31 \%$ and thus require further evaluation with biopsy [1]. We report six cases in which patients were found to have a complex solid and cystic mass, all of which were proven to be malignant breast cancers of varying etiologies. We also review the literature on malignant etiologies of complex solid and cystic breast masses, including their clinical presentation, work-up, histopathologic and immunochemistry findings, treatment, and prognosis.
\end{abstract}

\section{CASE SERIES}

\section{CASE SERIES}

\section{CASE 1:}

A 67-year-old female presented for diagnostic breast imaging. Two years prior, she was found to have a right breast simple cyst at an outside hospital (Figure 1). The patient presented to our facility complaining that the right breast cyst was progressively enlarging. General physical exam demonstrated a non-tender right breast lump.

Bilateral diagnostic mammogram showed a $7.2 \mathrm{~cm}$ partially imaged oval mass with obscured margins in the upper outer quadrant of the right breast, corresponding to the palpable abnormality (Figure 2). On ultrasound, there was an $8.8 \times 4.3 \times 7.5 \mathrm{~cm}$ oval, anechoic mass spanning from 8:0011:00 o'clock, $4 \mathrm{~cm}$ from the nipple, with indeterminate soft tissue demonstrating vascularity along its superficial and lateral margin, consistent with a complex solid and cystic mass (Figure 3). There was no axillary lymphadenopathy.

Subsequent ultrasound guided right breast cyst aspiration was performed to decrease its size prior to the core biopsy, as well as to send fluid cytology to evaluate for malignant cells. The cyst aspirate yielded $50 \mathrm{~mL}$ of dark maroon fluid, which was sent to cytology. Ultrasound-guided core needle biopsy of the solid component yielded infiltrating poorly differentiated ductal carcinoma, grade 3 of 3 (Nottingham score $=8$ ) in one core with the longest tumor extent in the core measuring 0.2 $\mathrm{cm}$ (Figure 4). Coincidentally, ductal carcinoma in situ, Van Nuys grade 3 of 3 , was demonstrated in two cores lining the fibrotic cyst wall. Tumor markers demonstrated a high proliferation index for Ki-67 (greater than 50\% invasive tumor nuclei stain), estrogen receptor negative, progesterone receptor negative, HER-2/neu negative and negative p-53 for overexpression. It is hypothesized that a cancer developed adjacent to the cyst, invaded the cyst wall and hemorrhaged into the cyst, expanding it. Clinical stage was difficult to determine due to the large cystic component of the tumor, but was favored to be T2 versus T3, N0 clinically. PET/CT demonstrated a hypermetabolic focus within the right breast corresponding to the patient's known malignancy, however was negative for evidence of regional or distant metastases (Figure 5). 
There was extensive discussion regarding the patient's treatment plan. Neoadjuvant chemotherapy was recommended since the tumor was triple negative and T2/T3. The patient completed 4 cycles of neoadjuvant chemotherapy with carboplatin/weekly paclitaxel.

A repeat diagnostic mammogram and ultrasound after the completion of neoadjuvant chemotherapy demonstrated a decrease in size of the right breast mass when compared to the baseline study (Figures 6 and 7). The patient was deemed a good candidate for breast conservation and ultimately opted for a right breast partial mastectomy and sentinel lymph node biopsy which showed residual infiltrating poorly differentiated duct carcinoma, grade 3 of 3 (Nottingham score $=9$ ) measuring $1.8 \times 1.6 \times 1.0 \mathrm{~cm}$ and a minor component of residual ductal carcinoma in situ, Van Nuys grade 3 of 3. There was no evidence of metastasis in $3 / 3$ lymph nodes. She has since recovered from treatment and is without clinical evidence of regional or distant recurrence.

\section{CASE 2:}

A 73-year-old female with history of a right breast simple cyst (Figures 8 and 9) and left breast infiltrating ductal carcinoma, with tumor markers demonstrating estrogen and progesterone receptor positivity and HER-2/neu negativity, status post left partial mastectomy with negative margins and 0/1 sentinel node with metastases, was noted to have a small palpable right breast lump upon completion of adjuvant radiation therapy. She had not yet started adjuvant endocrine therapy. Physical exam demonstrated a $1 \mathrm{~cm}$ firm, non-tender, slightly mobile nodule palpated near the chest wall at the upper outer quadrant, approximately $15 \mathrm{~cm}$ from the nipple. No axillary lymph nodes or skin changes were identified.

Diagnostic right breast mammogram demonstrated an oval, circumscribed mass in the upper outer quadrant of the right breast, posterior third depth, increased in size compared to the previous examination (Figure 10). On ultrasound, this mass corresponded to the known right breast cyst at the 12:00 axis, $10 \mathrm{~cm}$ from the nipple, which now demonstrated new vascular internal mural nodularity and measured $1.6 \mathrm{x} 1.1 \mathrm{x}$ $1.2 \mathrm{~cm}$ (Figure 11).

Subsequent ultrasound-guided core needle biopsy yielded infiltrating well-differentiated duct carcinoma, grade 1 of 3 (Nottingham score $=4$ ) in 3 cores, with the longest tumor extent in the core measuring $0.3 \mathrm{~cm}$. There was extensive intracystic ductal carcinoma in situ, cribriform type, Van Nuys grade 1 of 3 , in both blocks (Figure 12). Tumor markers demonstrated greater than $95 \%$ estrogen receptor positivity with an intermediate recurrence risk (Ki-67 index of 11-50\%), greater than $95 \%$ progesterone receptor positivity, HER-2/neu negative, and p-53 negative for overexpression.

The patient underwent needle localization, right partial mastectomy localization and sentinel node biopsy. The right breast partial mastectomy showed infiltrating well differentiated duct carcinoma, grade 1 of 3 (Nottingham score $=4$ ) measuring $0.5 \times 0.3 \times 0.3 \mathrm{~cm}$ in greatest dimension, and ductal carcinoma in situ, Van Nuys grade 1 of 3, cribriform pattern, measuring $0.6 \mathrm{~cm}$ in greatest dimension. The resection margins were free of tumor and there was no evidence of metastasis in the sentinel lymph node. The patient completed a course of radiation therapy to the right breast and is currently on anastrozole $1 \mathrm{mg}$ daily to complete a 5-year course.

\section{CASE 3:}

A 76-year-old female presented for diagnostic imaging after a $0.9 \mathrm{~cm}$ right breast nodule was incidentally found on computed tomography (CT) of the chest performed to evaluate for bronchiectasis (Figure 13). She had no breast complaints.

Bilateral diagnostic mammogram showed a round, circumscribed, hyperdense mass in the superior central right breast, corresponding to the CT finding (Figure 14). On ultrasound, this mass corresponded to a complex solid and cystic mass at the $12: 00$ axis, $15 \mathrm{~cm}$ from the nipple, measuring $1.1 \times 0.9 \times 1.1 \mathrm{~cm}$ (Figure 15).

Subsequent ultrasound-guided core needle biopsy yielded invasive ductal carcinoma, grade 2 of 3 (Nottingham score $=$ 7) with mucinous features in 2 of 3 cores (Figure 16). The longest tumor extent in a single core was $0.5 \mathrm{~cm}$ with background features suggestive of solid papillary carcinoma. Tumor markers demonstrated $95 \%$ estrogen receptor positivity, with an intermediate recurrence risk (Ki-67 index of $11-50 \%$ ), $70 \%$ progesterone receptor positivity, HER-2/neu negative, and p-53 negative for overexpression.

The patient underwent needle localization, partial right mastectomy and sentinel node biopsy. The right breast partial mastectomy showed infiltrating moderately differentiated duct carcinoma, grade 2 of 3 (Nottingham score $=7$ ), with mucinous features measuring $0.7 \times 0.4 \mathrm{~cm}$ (Figure 17). The resection margins were free of tumor, with the closest clearance of $0.3 \mathrm{~cm}$ at the anterior margin. There was no evidence of metastasis in $6 / 6$ lymph nodes. She is currently receiving adjuvant breast radiation therapy with plans to complete at least a 5-year course of adjuvant endocrine therapy with anastrozole $1 \mathrm{mg}$ daily.

\section{CASE 4:}

A 52-year-old female with several month history of right breast non-bloody discharge and palpable right breast lump initially presented to an outside hospital where she was found to have two complex solid and cystic masses of the right breast at the 6:00 axis and the 8:00 axis, respectively (Figures 18 and 19). The complex solid and cystic mass at the 6:00 axis was biopsied at the outside hospital with pathology consistent with at minimum ductal carcinoma in-situ, Van Nuys grade 2 of 3, cribriform type, involving 15 fragments of intraductal papilloma and serum markers demonstrating greater than $80 \%$ estrogen and progesterone receptor positivity.

The patient was subsequently referred to our institution where she underwent ultrasound-guided core needle biopsy of the right breast mass at the 8:00 axis, $4 \mathrm{~cm}$ from the nipple. Biopsy results yielded ductal carcinoma in-situ, Van Nuys grade 1 of 3, solid and cribriform types (Figure 20). 
Right breast total mastectomy and sentinel lymph node biopsy were performed which demonstrated ductal carcinoma in-situ, Van Nuys grade 2 of 3, cribriform type in 3 blocks with the largest extent in a single block measuring $0.7 \mathrm{~cm}$ (Figure 21). The resection margins were free of tumor. There was no evidence of metastasis in 2 lymph nodes. Tumor markers demonstrated greater than $95 \%$ estrogen receptor positivity and p53 negative for over-expression. Further discussions regarding adjuvant endocrine therapy are planned after the patient has fully recovered from surgery.

\section{CASE 5:}

A 62-year-old male, with no family history of breast cancer, who has been undergoing testosterone supplementation for the past 2 years for central hypogonadism, presented for diagnostic breast imaging. He complained of a palpable, painless right breast lump for the past 4 months.

Diagnostic right breast mammogram demonstrated a circumscribed, oval, hyperdense mass in the subareolar region corresponding to the palpable area of concern (Figure 22). On ultrasound, this mass corresponded to a complex solid and cystic mass in the subareolar region measuring $3.5 \times 3.1 \times 2.9$ $\mathrm{cm}$ with increased vascularity within the solid components of the mass (Figure 23).

Subsequent ultrasound-guided core needle biopsy yielded at minimum ductal carcinoma in-situ, Van Nuys grade 2 of 3, papillary and cribriform types, associated with an atypical papillary proliferation with features suggestive of encapsulated papillary carcinoma (Figure 24). Tumor markers demonstrated $95 \%$ estrogen receptor positivity with an intermediate recurrence risk (Ki-67 index of 11-50\%), 95\% progesterone receptor positivity, HER-2/neu negative, and p53 negative for overexpression.

It was recommended that the patient discontinue testosterone therapy as some breast cancers express androgen receptors. He underwent genetic testing and was found to be negative for a number of pathogenic mutations, including BRCA1, BRCA1, AND PALB2.

A total right mastectomy and sentinel node biopsy was performed, revealing encapsulated papillary carcinoma, measuring $2.4 \mathrm{~cm}$ (Figure 25). The resection margins were free of tumor and there was no evidence of metastasis in $3 / 3$ lymph nodes. The patient was considered to be very low risk for locoregional recurrence or for contralateral primary breast cancer, given that he is a male with negative BRCA testing. Accordingly, systemic adjuvant therapy was not recommended.

\section{CASE 6:}

A 47-year-old female presented for diagnostic breast imaging. Seven months prior, she underwent routine screening mammogram, which was negative for suspicious findings (Figure 26). The patient returned for diagnostic imaging seven months later and complained of a right breast lump that she initially palpated one month after her screening exam and that had progressively enlarged over 6 months.

Diagnostic right breast mammogram demonstrated a new, circumscribed, round mass in the lower outer quadrant of the right breast, anterior and middle third depth, corresponding to the palpable area of concern (Figure 27). On ultrasound, this mass corresponded to an oval, complex solid and cystic mass with focally indistinct margins and hypervascular solid component, at the 7:00-8:00 axis, $4 \mathrm{~cm}$ from the nipple, measuring $4.9 \times 3.1 \times 4.9 \mathrm{~cm}$ (Figure 28). A round, solid mass with indistinct margins was also found at the 10:00 axis, $4 \mathrm{~cm}$ from the nipple, measuring $0.8 \times 0.5 \times 0.6 \mathrm{~cm}$. This mass was located $1.5 \mathrm{~cm}$ from the dominant lesion and was suspicious for a satellite lesion.

Subsequent ultrasound-guided core needle biopsy of the dominant lesion yielded infiltrating mammary carcinoma with focal features suggestive of metaplastic carcinoma (osteoclast giant cells and focal squamous metaplasia), (Nottingham score $=7$ ) in 4 cores with the longest tumor extent in a single core measuring $2.0 \mathrm{~cm}$ with some necrosis (Figure 29). Additionally, ductal carcinoma in-situ, Van Nuys grade 2 of 3, solid type was found in 2 cores with the longest extent in a single core measuring $0.2 \mathrm{~cm}$. Tumor markers demonstrated 95\% estrogen receptor positivity (Figure 30) with an intermediate recurrence risk by Ki-67 index (11-50\% invasive tumor nuclei stain), $95 \%$ progesterone receptor positivity, HER-2/neu negative, and p-53 negative for overexpression.

The patient is currently awaiting biopsy of a smaller, solid, suspected satellite lesion with clip placement to determine if she is a candidate for breast conserving therapy. She will undergo neoadjuvant chemotherapy prior to surgery.

\section{DISCUSSION}

\section{Etiology \& Demographics:}

Complex solid and cystic masses can occur at almost any age in females and occasionally in males due to the broad range of benign and malignant etiologies, but most are commonly seen in women ages 30-50 [1-2]. Complex solid and cystic masses typically arise when a solid mass gradually fills and dilates a lactiferous duct. When the duct is sufficiently dilated, the mass appears as a complex solid and cystic lesion on ultrasound, as described by A. Athanasiou et al [3]. Occasionally, solid malignant masses can secrete mucin or fluid or undergo necrosis, which can also result in complex solid and cystic morphology.

\section{Clinical \& Imaging Findings:}

Complex solid and cystic masses are defined as a cyst with thick walls $(>0.5 \mathrm{~mm})$, thick septa $(>0.5 \mathrm{~mm})$, an intracystic mass, or a cyst demonstrating $>50 \%$ solid components. A study by Houssami N., et al found that complex solid and cystic masses were estimated to be reported in approximately $5 \%$ of breast ultrasound examinations [4]. 
Complex solid and cystic masses fall into BIRADS category 4 and require further evaluation with biopsy. Doshi et al. characterized the malignant potential of complex solid and cystic masses to be between 23 and $31 \%$, underlying the need for biopsy, typically core needle targeting the solid component, but surgical excision can also be considered when appropriate. Fine needle aspiration of the cystic component is generally avoided to ensure identification of the solid component during biopsy [1]. Upon completion of the biopsy, a localization clip should be deployed at the biopsy site for future reference. If the lesion is determined to be benign and concordant with imaging, follow up with clinical breast exams and possible breast imaging studies at 6- or 12-month intervals can be performed to ensure two-year stability of the lesion [5]. In addition to core needle and surgical biopsy, a vacuumassisted complete excision of the complex solid and cystic mass is sometimes performed for definitive treatment [6].

\section{Treatment \& Prognosis:}

Treatment of complex solid and cystic masses is varied. It is based on the immunohistochemical subtype of the tumor cells, as opposed to the complex solid and cystic imaging appearance. Prognosis is also based on the immunohistochemical profile. Information regarding treatment and prognosis by tumor subtype is described in greater detail below.

\section{Differential Diagnosis:}

The differential for complex solid and cystic masses is broad and includes both benign and malignant etiologies. Benign complex solid and cystic masses can include such processes as cysts with confluent debris, galactoceles, papillomas, abscesses, hematomas, granulomatous inflammation and fat necrosis. Less commonly, fibroadenomas and phyllodes tumors can present with complex solid and cystic morphology. Atypical lesions, such as atypical ductal hyperplasia, atypical lobular hyperplasia, lobular carcinoma in situ and atypical papillomas can also present with this appearance. Per Doshi et al., invasive ductal carcinoma and ductal carcinoma in situ are the most likely malignant breast tumors to present as complex solid and cystic masses on ultrasound [1].

The above cases all demonstrated sonographic morphology of complex solid and cystic masses and were found to be malignant lesions of varying etiologies including invasive ductal carcinoma, ductal carcinoma in situ, mixed mucinous and papillary carcinoma, mucinous carcinoma, ductal carcinoma in situ with intraductal papilloma, and papillary carcinoma, including one case in a male patient. The most common malignant etiologies are discussed in greater detail below.

\section{Invasive ductal carcinoma}

Invasive ductal carcinoma (IDC) comprises approximately $80 \%$ of all breast malignancies, with a median age of diagnosis of 62 [7]. Numerous risk factors have been identified including early age of menarche, late age of menopause, nulliparity, obesity, non-Hispanic white race, failure to breastfeed, BRCA status, and estrogen levels [7-10]. In the United States, most cases of IDC are found during routine screening mammography, but up to $15 \%$ of cases are discovered due to the presence of a palpable breast mass [11].

On mammography, IDC typically appears as an irregularly shaped mass with spiculated margins, oftentimes accompanied by segmentally distributed pleomorphic microcalcifications. Sonographically, IDC most often appears as an irregular hypoechoic mass that is not parallel, with angular or spiculated margins, and demonstrates posterior acoustic shadowing [12]. IDC can occasionally present as a complex solid and cystic mass, which is usually secondary to necrosis.

If the lesion in question is categorized as BIRADS 4 or 5, a core needle biopsy should be performed as it allows for a definitive diagnosis more often than fine-needle aspiration [13]. If a cystic component is present, vacuum assisted core needle biopsy may be preferred [6]. The American Society of Clinical Oncology/College of American Pathologists recommend routine testing of newly diagnosed invasive breast cancer for ER/PR/HER2 receptor expression [14]. As with other breast cancers, assessment of spread to local and regional lymph nodes is necessary to stage the tumor.

IDC arises from epithelial cells of abnormal lobular units and is classified into several histological subtypes including apocrine, mucinous, papillary, tubular and others, however, $75 \%$ of IDC displays no specific morphological features, and are classified as IDC not otherwise specified (NOS) or "no special type" (NST) [15].

In a study of 824 cases of IDC, most specimens were $\mathrm{ER}+/ \mathrm{PR}+$ and HER2 - (65\%), with triple negative expression occurring in $15 \%$ of cases [16].

Surgical excision is the primary treatment for IDC. Both mastectomy or breast conservation therapy are viable options, and the choice depends on the stage, size and margins of the cancer, as well as personal preferences. Breast conservation typically involves adjuvant radiation therapy. Hormonal therapy is well validated for tumors expressing endocrine receptors. Likewise, trastuzumab is proven effective if HER2 proteins are expressed on the tumor. With regards to chemotherapy, in a study of 1134 invasive breast cancers, including 824 cases of IDC, chemotherapy improved diseasefree and overall survival, though the significance of the improvement depended on hormone receptor expression [16].

Prognosis depends heavily on the receptor status of the tumor, with triple negative carcinoma conferring the worst prognosis. Triple negative IDC has an approximate 5-year survival of $70 \%$ and disease-free survival of $74 \%$. ER+/PR+, HER2- tumors had the best prognosis with an approximate 5year survival of $90 \%$ and disease-free survival of $87 \%$. ER expression was shown to have the greatest impact on overall and disease-free survival (with positivity improving survival) [16]. 


\section{Ductal carcinoma in-situ}

Ductal carcinoma in situ (DCIS) incidence rates increase with age, and peak at ages 70-79. Risk factors for DCIS are similar to those for invasive cancer, and include family history of breast cancer, increased breast density, nulliparity or late age at first birth, obesity, and BRCA 1/BRCA 2 mutations [9]. The most common presentation of DCIS is an asymptomatic patient with no signs or symptoms of disease. DCIS is typically found as an abnormal mammogram finding, most often as microcalcifications or occasionally as architectural distortion. DCIS may, however, present as a palpable mass, nipple discharge, or Paget's disease [17]

DCIS may be calcified or noncalcified. On sonography, calcified DCIS most often appears as echogenic foci located within a mass or a duct, associated with internal microlobulations, or distributed in a branch pattern. Noncalcified DCIS, which is more often identified in symptomatic patients, may appear as a hypoechoic mass with microlobulated margins and no posterior acoustic features, or it may be a "pseudomicrocystic" appearance [18]. DCIS can less frequently present as an intracystic mass, sometimes associated with papillomas.

Definitive diagnosis is obtained via core needle or excisional biopsy, rather than fine-needle aspiration which often cannot distinguish between invasive and in situ disease due to lack of cytological architecture of the sample. Key histopathological findings include the grade, whether margins were involved, whether the lesion expresses estrogen and progesterone receptors, and the cytoarchitectural features/ growth pattern (including comedo type, cribriform type, micropapillary type, papillary type, and solid type) [19].

Estrogen and progesterone receptor expression are important prognostic indicators and help guide therapy of DCIS. Aromatase inhibitors and tamoxifen are approved to reduce recurrence rates of DCIS. Testing for HER2 expression is not currently indicated for DCIS in accordance with the American Society of Clinical Oncology, as therapy directed at HER2 receptors have not proven to improve outcomes in DCIS [20].

Treatment of DCIS typically involves mastectomy or breast conservation therapy. Depending on the immunochemistry, aromatase inhibitors may improve outcomes [19]. Chemotherapy has no role in the treatment of DCIS due to the low metastatic potential with appropriate treatment.

Prognosis of DCIS with appropriate treatment in excellent. According to Narod, SA et al., an analysis of the Surveillance, Epidemiology, and End Results (SEER) database revealed that the 20 -year breast cancer mortality among women with DCIS was 3.3 percent. The risk of invasive recurrence at 20 years was also favorable, at 5.9 and 6.2 percent, involving the ipsilateral and contralateral breast, respectively [21].

\section{Mucinous carcinoma}

Mucinous carcinoma comprises approximately $2 \%$ of all invasive breast cancers and is a subtype of invasive ductal carcinoma. According to a study of 25 patients with this lesion type, the average age at diagnosis was 62.3 [22]. Mucinous carcinoma that is not identified on screening mammograms, presents most commonly with a palpable breast mass $(94.8 \%)$ or less commonly nipple discharge (1.9\%) [23]. A study by Lam et al found that mucinous carcinoma commonly appeared as a mass with microlobulated or circumscribed margins on mammography, and as a complex mass with cystic and solid components, vascularity, and distal enhancement on sonography [24]. Further evaluation with core needle or excisional biopsy is usually warranted. Histologically, pure mucinous carcinomas appear as paucicellular, with a $90 \%$ mucoid component.

A study of 309 patients with pure mucinous carcinomas found that estrogen and progesterone receptors are more likely to be overexpressed when compared to other invasive ductal carcinomas, meaning hormonal therapy is likely to be effective. The same study found that HER2 expression is decreased compared to other IDCs [23].

Similar to other invasive breast cancers, treatment of mucinous carcinoma involves surgical resection, either mastectomy or lumpectomy with radiation therapy, along with hormonal and chemotherapy.

The prognosis of mucinous carcinoma is significantly better than IDC of no special type, with a 10-year survival of over $90 \%$ [25]. Another study of 268 cases of mucinous carcinoma found that the disease-free survival is $95.2 \%$ after 5 years, as opposed to $92.0 \%$ for IDC not otherwise specified [26].

\section{Intracystic papillary carcinoma}

Intracystic papillary carcinoma (IPC) is a rare entity, with limited data available. One study of 917 cases of IPC revealed an average age of onset of 69.5 years, affecting predominantly Caucasian women with a higher percentage of cases in men than other breast cancers. [27] IPC usually presents clinically with a palpable breast mass $(80 \%)$ and/ or nipple discharge $(15 \%)$ in a post-menopausal woman [27].

Per Doshi et al., intracystic papillary carcinomas present as complex sold and cystic masses in $24-57 \%$ of cases [1]. Although mammographic findings are nonspecific, IPC usually appears as a round or oval shaped mass with microlobulated or circumscribed margins, most often in the retroareolar region [28]. Work-up of IPC involves pathological confirmation via excisional or core needle biopsy. Fine needle aspiration is typically avoided as it is often incapable of distinguishing malignant papillary carcinomas from benign papillomas [29]. Vacuum assistance is often recommended to improve sample yield [3]. The architectural appearance of IPC is usually either papillary (41\%), cribriform (35\%), or solid (24\%). Cells appear uniformly round, columnar, or spindle shaped, without myoepithelial or benign apocrine cells [30]. 
In one study of 29 cases of IPC, all had estrogen receptor overexpression, and none expressed HER2. Ki-67 data was available for 20 of the cases, of which 12 demonstrated intermediate recurrence risk (Ki-67 index 11-50\%) [30].

Surgery is the mainstay of treatment, with breast conserving therapy having equal recurrence rates to mastectomy [29]. Radiation therapy in conjunction with lumpectomy did not have a significant impact on recurrence or survival. There is little data to support the use of chemotherapy or hormonal therapy in IPC, although they are frequently employed [29].

Overall survival of IPC is $82 \%$ and $70 \%$ at 5- and 10years respectively, compared to $75 \%$ and $56 \%$ for other invasive breast cancers. Likewise, disease free survival in IPC is $85 \%$ and $77 \%$, indicating a better prognosis for IPC. [27, 31]

This case series focuses on complex solid and cystic masses that were all found to be malignant, however widely varied in their specific etiologies and presentations. In these cases, recognition of thickened or nodular walls and of solid components of cysts led to biopsy and pathological analysis in each patient, which in turn ultimately led to the diagnoses of malignancy. As prior studies have shown that $23-31 \%$ of complex solid and cystic masses are malignant [1], continued vigilance is necessary on the part of the diagnostic radiologist to identify these malignant lesions.

\section{TEACHING POINT}

A cyst containing a thick wall, internal septations, or a solid intracystic component is defined as a complex solid and cystic breast mass. These lesions carry a malignant potential between $23-31 \%$ and thus require further evaluation with ultrasoundguided core needle biopsy.

\section{REFERENCES}

1. Doshi DJ, March DE, Crisi GM, Coughlin BF. Complex cystic breast masses: diagnostic approach and imagingpathologic correlation. Radiographics 2007;27(Suppl 1):S53S64. PMID: 18180235.

2. Hines N, Slanetz PJ, Eisenberg RL. Cystic masses of the breast. AJR Am J Roentgenol. 2010; 194(2):122-133.

3. Athanasiou A, Aubert E, Vincent Salomon A, Tardivon A. Complex cystic breast masses in ultrasound examination. Diagn Interv Imaging. 2014; 95:169-179. PMID: 24433921.

4. Houssami N, Irwig L, Simpson JM, McKessar M, Blome S, Noakes J. The Sydney Breast Imaging Accuracy Study: comparative sensitivity and specificity of mammography and sonography in women with symptoms. Am. J. Roentgenol. 2003; 180:935?40. PMID: 12646432.
5. Bevers TB, Helvie M, Bonaccio E, et. al. Breast Cancer Screening and Diagnosis (Version 3.2018). JNCCN. 2018; 16(11): 1368 .

6. Quinn-Laurin V, Hogue JC, Pinault S, Duchesne N. Vacuum-assisted complete excision of solid intraductal/intracystic masses and complex cysts: Is follow-up necessary? Breast. 2017; 35:42-47. PMID: 28646723.

7. American Cancer Society. Breast Cancer Facts \& Figures 2017-2018. Atlanta: American Cancer Society, Inc. 2017; 3.

8. Key T, Appleby P, Barnes I, Reeves G, Endogenous Hormones and Breast Cancer Collaborative Group. Endogenous sex hormones and breast cancer in postmenopausal women: reanalysis of nine prospective studies. J Natl Cancer Inst. 2002; 94(8):606. PMID: 11959894.

9. Lauby-Secretan B, Scoccianti C, Loomis D, Grosse Y, Bianchini F, Straif K, International Agency for Research on Cancer Handbook Working Group. Body Fatness and Cancer-Viewpoint of the IARC Working Group. N Engl J Med. 2016; 375(8):794. PMID: 27557308.

10. Kumar V, Abbas AK, Aster JC. Robbins and Cotran pathologic basis of disease (Ninth edition.). Philadelphia, PA: Elsevier/Saunders. 2015: 1047-1052.

11. Esserman LJ, Shieh Y, Rutgers EJ, et al. Impact of mammographic screening on the detection of good and poor prognosis breast cancers. Breast Cancer Res Treat. 2011; 130(3):725-34. PMID: 21892702.

12. Yang WT, Hennessy $B$, Broglio $K$, et al. Imaging differences in metaplastic and invasive ductal carcinomas of the breast. AJR Am J Roentgenol. 2007; 189(6):1288-93. PMID: 18029860.

13. ?ukasiewicz E, Ziemiecka A, Jakubowski W, Vojinovic J, Bogucevska M, Dobruch-Sobczak K. Fine-needle versus coreneedle biopsy - which one to choose in preoperative assessment of focal lesions in the breasts? Literature review. J Ultrason. 2017; 17(71): 267-274. PMID: 29375902.

14. Wolff AC, Hammond ME, Schwartz JN, et al. American Society of Clinical Oncology/College of American Pathologists guideline recommendations for human epidermal growth factor receptor 2 testing in breast cancer. J Clin Oncol. 2007; 25(1):118-45. PMID: 17159189.

15. Lakhani SR, Ellis IO, Schnitt SJ, Tan PH, Van de Vijver MJ, editors. WHO Classification of Tumours of the Breast. Fourth ed. IARC; Lyon: 2012: 252.

16. Onitilo AA, Engel JM, Greenlee RT, Mukesh BN. Breast Cancer Subtypes Based on ER/PR and Her2 Expression: Comparison of Clinicopathologic Features and Survival. Clin Med Res. 2009; 7(1-2):4-13. PMID: 19574486.

17. Günhan-Bilgen I, Oktay A. Paget's disease of the breast: clinical, mammographic, sonographic and pathologic findings 
in 52 cases. Eur J Radiol. 2006; 60(2):256-63. PMID: 16887314

18. Wang LC, Sullivan M, Du H, Feldman MI, Mendelson EB. US appearance of ductal carcinoma in situ. Radiographics. 2013; 33(1):213-28. PMID: 23322838.

19. Fisher B, Dignam J, Wolmark N, et al. Tamoxifen in treatment of intraductal breast cancer: National Surgical Adjuvant Breast and Bowel Project B-24 randomised controlled trial. Lancet. 1999; 353(9169):1993. PMID: 10376613.

20. National Comprehensive Cancer Network Guidelines. Breast Cancer (Version 4.2018) Ductal Carcinoma in Situ (DCIS).

https://www.nccn.org/professionals/physician_gls/pdf/breast.p df. Accessed on February 14, 2019.

21. Narod SA, Iqbal J, Giannakeas V, Sopik V, Sun P. Breast Cancer Mortality After a Diagnosis of Ductal Carcinoma In Situ. JAMA Oncol. 2015; 1(7):888. PMID: 26291673.

22. Dumitru A, Procop A, Iliesiu A, et al. Mucinous Breast Cancer: a Review Study of 5 Year Experience from a Hospital-Based Series of Cases. Maedica. 2015; 10(1):14-18. PMID: 26225144.

23. Cao AY, He M, Liu ZB, et al. Outcome of pure mucinous breast carcinoma compared to infiltrating ductal carcinoma: a population-based study from China. Ann Surg Oncol. 2012; 19:3019-3027. PMID: 27232881.

24. Lam WW, Chu WC, Tse GM, et al. Sonographic appearance of mucinous carcinoma of the breast. AJR Am J Roentgenol. 2004; 182(4):1069-1074. PMID: 15039190.

25. Li CI. Risk of mortality by histologic type of breast cancer in the United States. Horm Cancer. 2010; 1:156-65. PMID: 21761358 .

26. Bae SY, Choi MY, Cho DH, Lee JE, Nam SJ, Yang JH. Mucinous Carcinoma of the Breast in Comparison with Invasive Ductal Carcinoma: Clinicopathologic Characteristics and Prognosis. J Breast Cancer. 2011; 14(4): 308-313. PMID: 22323918.

27. Grabowski J, Salzstein SL, Sadler GR, Blair S. Intracystic papillary carcinoma: a review of 917 cases. Cancer. 2008; 113:916. PMID: 18661510

28. Rodriguez $\mathrm{MCH}$, Secades AL, Angulo JM. Intracystic Papillary Carcinoma of the Breast. AFIP Archives - Best Cases from the AFIP. RadioGraphics. 2010; 30(7): 20212027.

29. Jeffrey PB, Ljung BM. Benign and malignant papillary lesions of the breast. A cytomorphologic study. Am J Clin Pathol. 1994; 101:500-507.

30. Leal C, Costa I, Fonseca D, et al. Intracystic (encysted) papillary carcinoma of the breast: a clinical, pathological, and immunohistochemical study. Hum Pathol. 1998; 29:10971104. PMID: 9781648.

31. Solorzano CC, Middleton LP, Hunt KK, et al. Treatment and outcome of patients with intracystic papillary carcinoma of the breast. Am J Surg. 2002; 184:364-368. PMID: 12383904. 


\section{FIGURES}

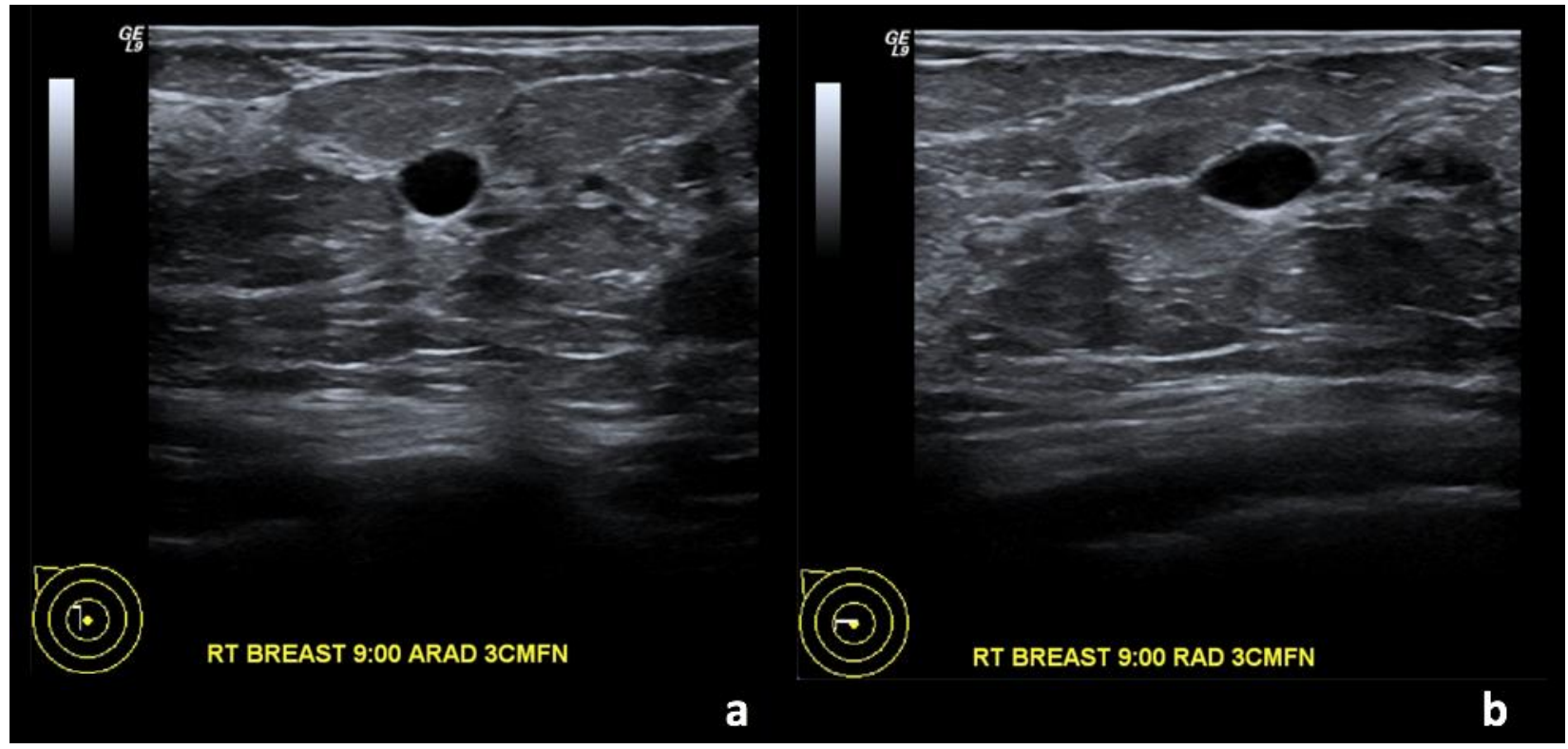

Figure 1: 67-year-old female with right breast invasive ductal carcinoma and ductal carcinoma in-situ. Two years prior to Figures 2 and 3.

Findings: (a, b) The right breast demonstrates an oval, circumscribed, parallel, anechoic mass with posterior acoustic enhancement at the 9 o'clock position, $3 \mathrm{~cm}$ from the nipple, measuring $0.8 \times 0.5 \times 0.4 \mathrm{~cm}$, consistent with a simple cyst.

Technique: Outside hospital ultrasound examination using a high frequency linear transducer (14 MHz).

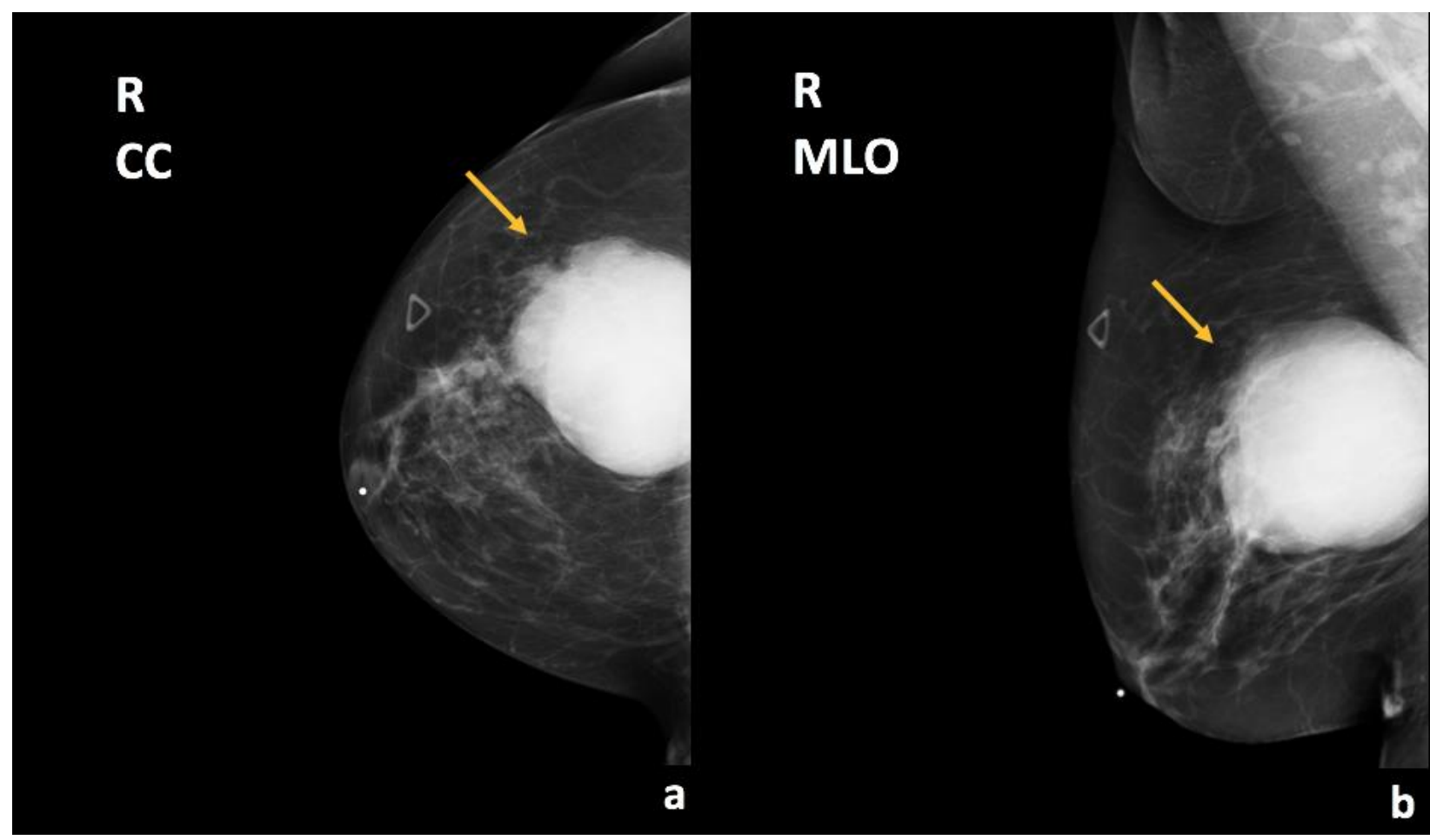

Figure 2: 67-year-old female with right breast invasive ductal carcinoma and ductal carcinoma in-situ.

Findings: There is a partially imaged oval mass with obscured margins in the upper outer quadrant (arrows), corresponding to the patient's right breast enlarging palpable abnormality.

Technique: Diagnostic mammogram (30 kVp, $78 \mathrm{mAs}$ ). (a) Craniocaudal (CC) and (b) mediolateral oblique (MLO) views of the right breast. 


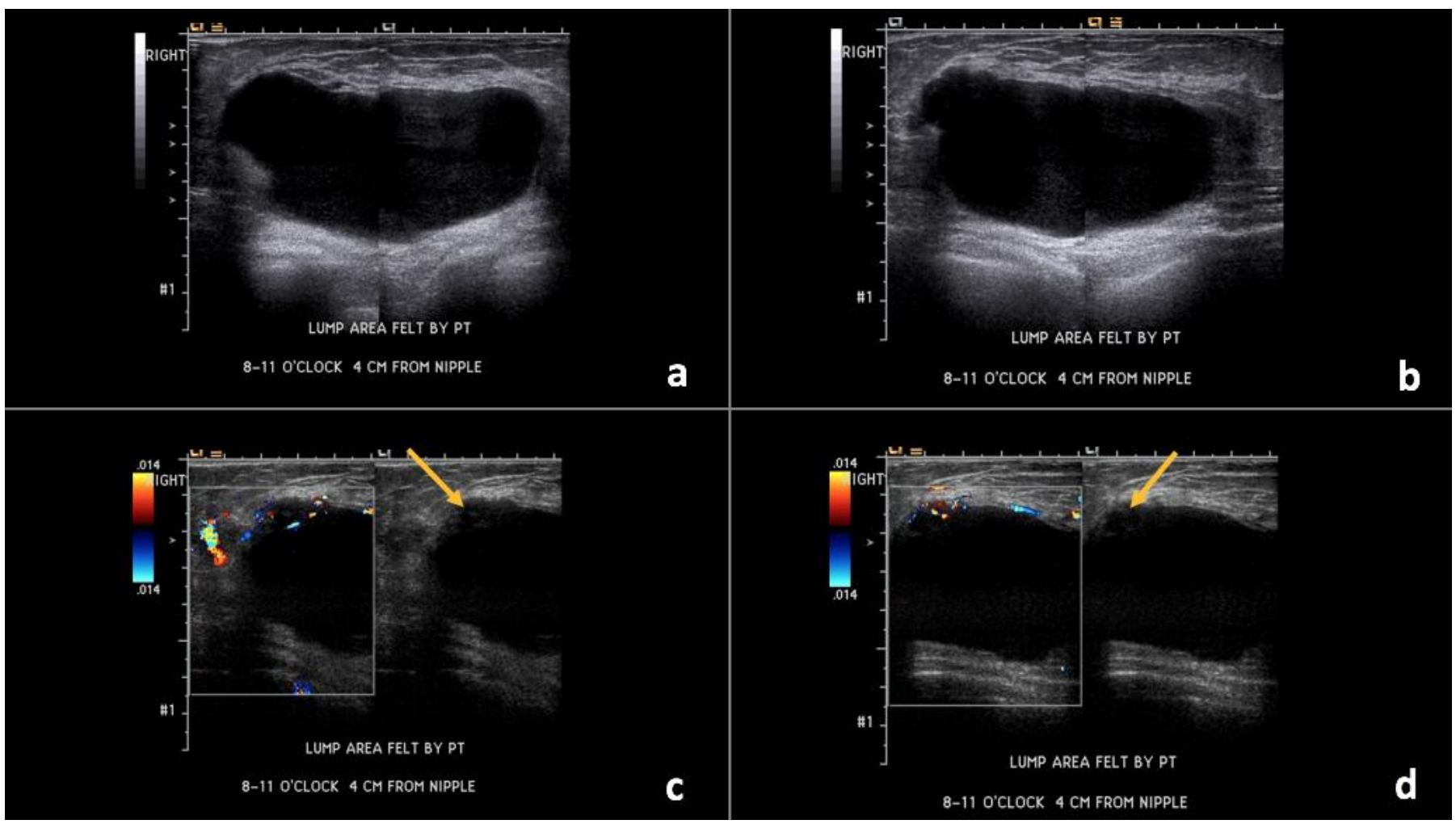

Figure 3: 67-year-old female with right breast invasive ductal carcinoma and ductal carcinoma in-situ.

Findings: (a, b) The right breast demonstrates an oval, circumscribed, parallel, predominately anechoic mass with minimal layering debris between the 8 o'clock to 11 o'clock position, $4 \mathrm{~cm}$ from the nipple, measuring $8.8 \times 4.3 \mathrm{x} 7.5 \mathrm{~cm}$. (c, d) Indeterminate soft tissue demonstrating vascularity is seen along the superficial and lateral margin of this mass (arrows), consistent with a complex solid and cystic mass.

Technique: Ultrasound and Color Doppler using a high frequency linear transducer (14 MHz).

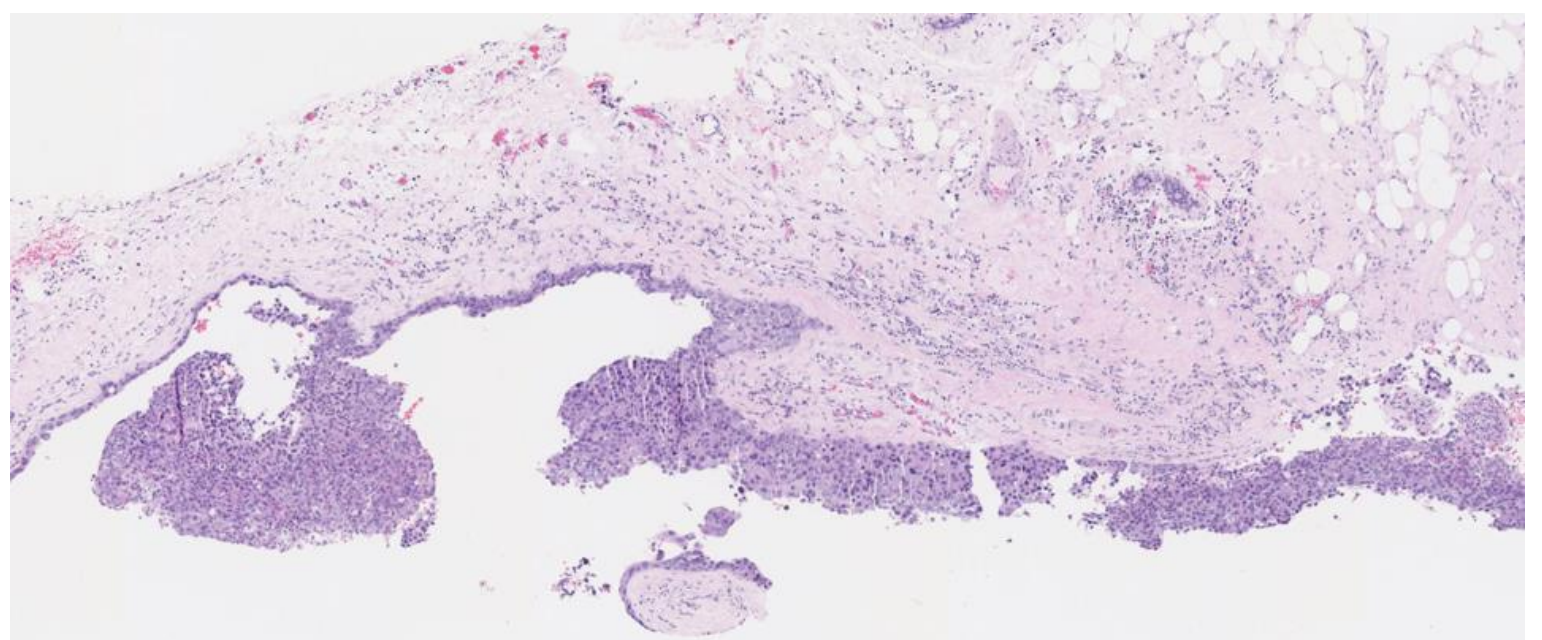

Figure 4: 67-year-old female with right breast invasive ductal carcinoma and ductal carcinoma in-situ.

Findings: The cystic lesion demonstrates infiltrating poorly differentiated duct carcinoma, grade 3 of 3 , Nottingham score of 8 . The tumoral cells exhibit increased mitosis, nuclear pleomorphism and no tubule formation, indicating high grade malignancy.

Technique: Microscopic examination with Hematoxylin and Eosin (H\&E) stain. Medium-power view (55x) from the core needle biopsy of a right breast complex solid and cystic mass. 


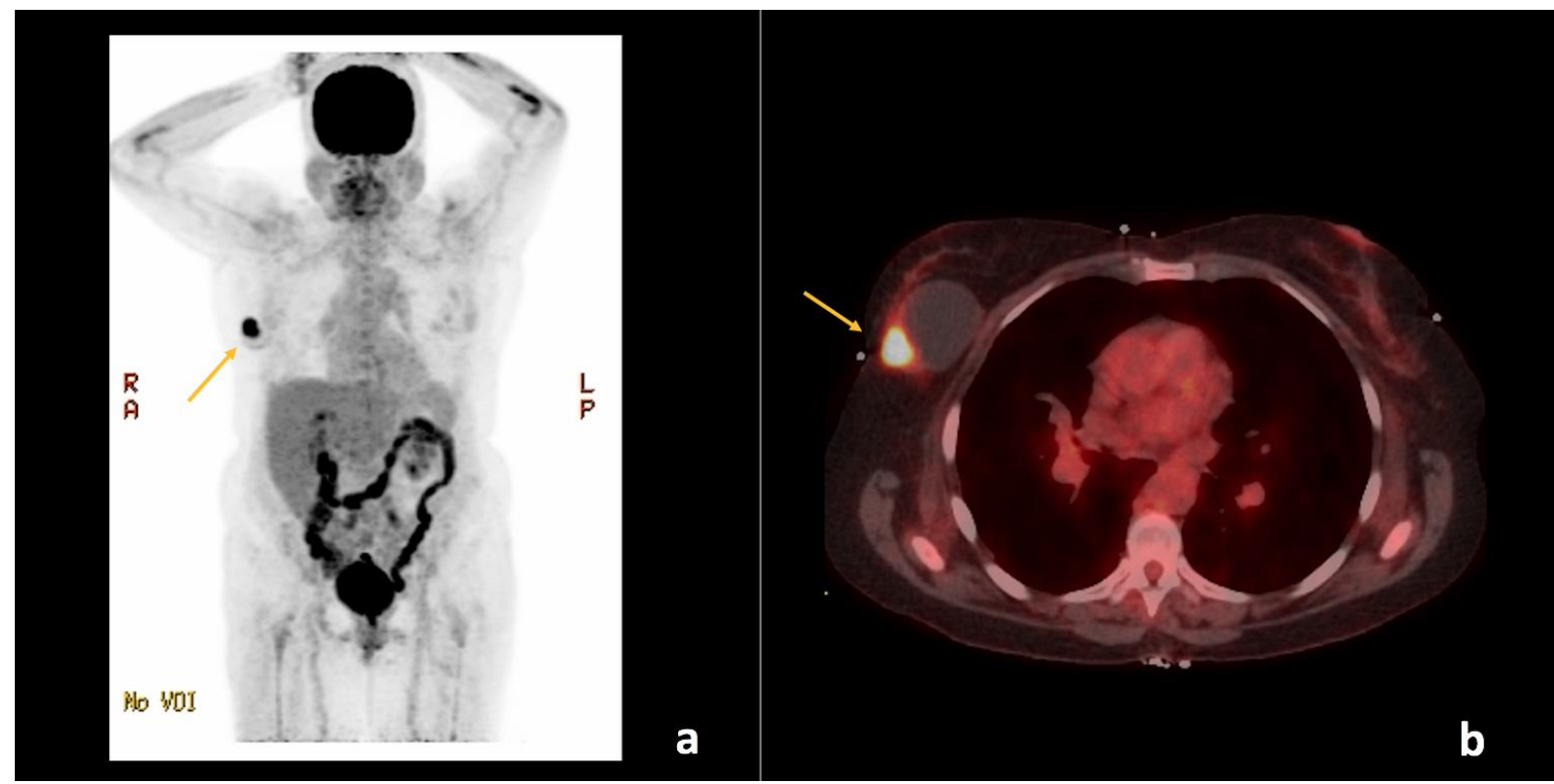

Figure 5: 67-year-old female with right breast invasive ductal carcinoma and ductal carcinoma in-situ.

Findings: There is a hypermetabolic focus within the right breast with maximum standardized uptake value (SUVmax) of 13.7 (arrows), corresponding to the patient's known primary malignancy. No evidence of regional or distant metastases.

Technique: Positron emission tomography-computed tomography (PET/CT) from the skull to the mid-thigh level. (a) Maximum intensity projection (MIP) and (b) Fused axial plane images through the upper chest approximately 67 minutes following intravenous injection of $12.268 \mathrm{mCi}$ of 18F-2-fluoro-2-deoxyglucose (F18-FDG) through the patient's right antecubital vein. The patient's fasting blood glucose measured just before intravenous injection was $75 \mathrm{mg} / \mathrm{dL}$. The low-dose CT was with oral contrast, but without intravenous contrast and was used for image fusion, attenuation correction, and lesion localization only. The standardized uptake values (SUVs) are normalized to the patient's body weight and indicate the highest activity concentration (SUVmax) in a given disease site. (GE Medical Systems, Discovery 690; $140 \mathrm{kVp}, 18 \mathrm{~mA}, 3.75 \mathrm{~mm}$ slice thickness. DFOV of MIP: 65.0 x $130.0 \mathrm{~cm}$. DFOV of Fused Axial image: $50.0 \mathrm{~cm})$.

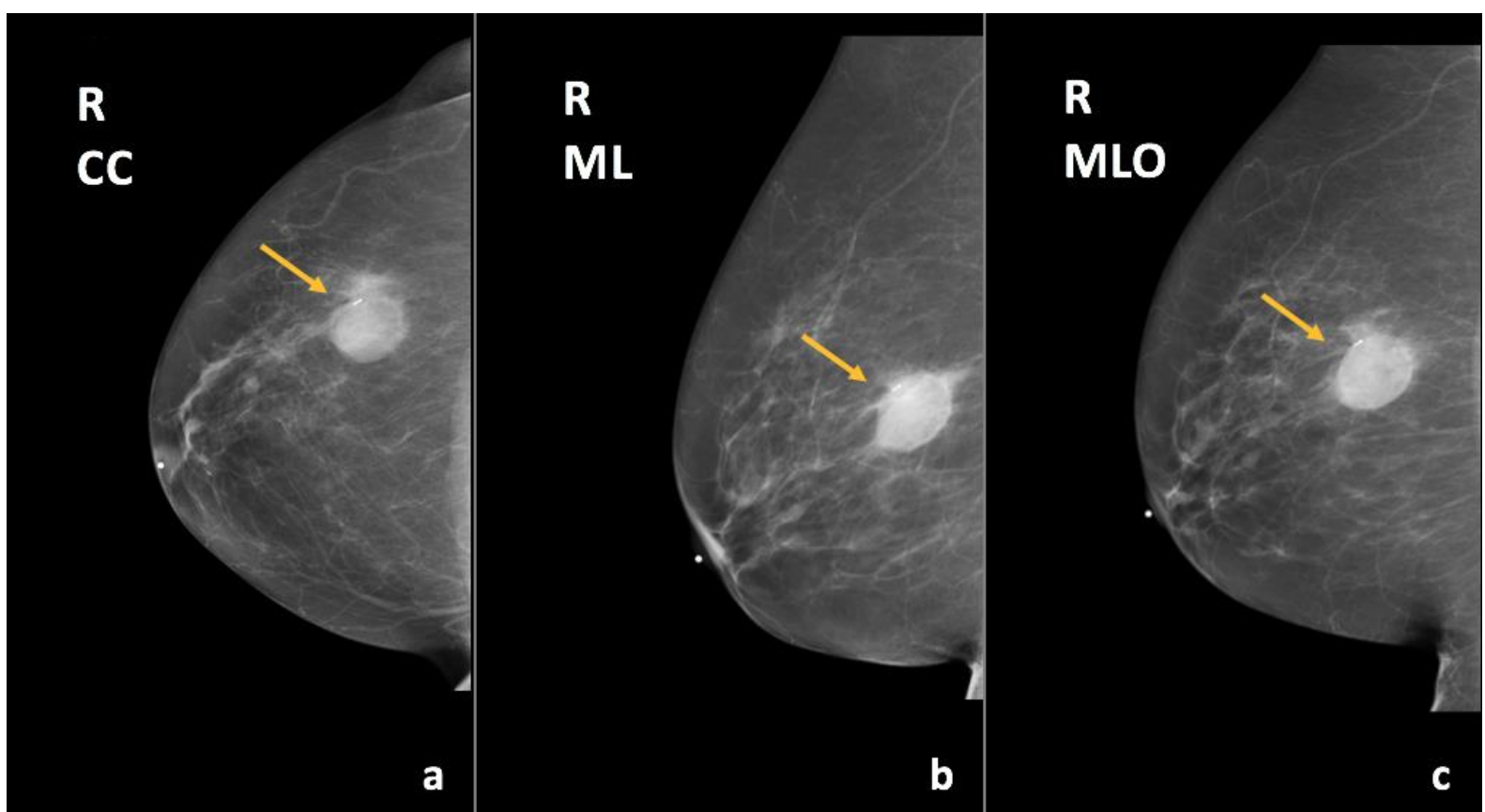

Figure 6: 67-year-old female with right breast invasive ductal carcinoma and ductal carcinoma in-situ.

Findings: There has been interval decreased size of the known mass in the upper outer quadrant of the right breast (arrows), containing a localization marker.

Technique: Diagnostic mammogram (29 kVp, $45 \mathrm{mAs}$ ) after completion of neoadjuvant chemotherapy. (a) Craniocaudal (CC), (b) mediolateral (ML), and (c) mediolateral oblique (MLO) views of the right breast. 


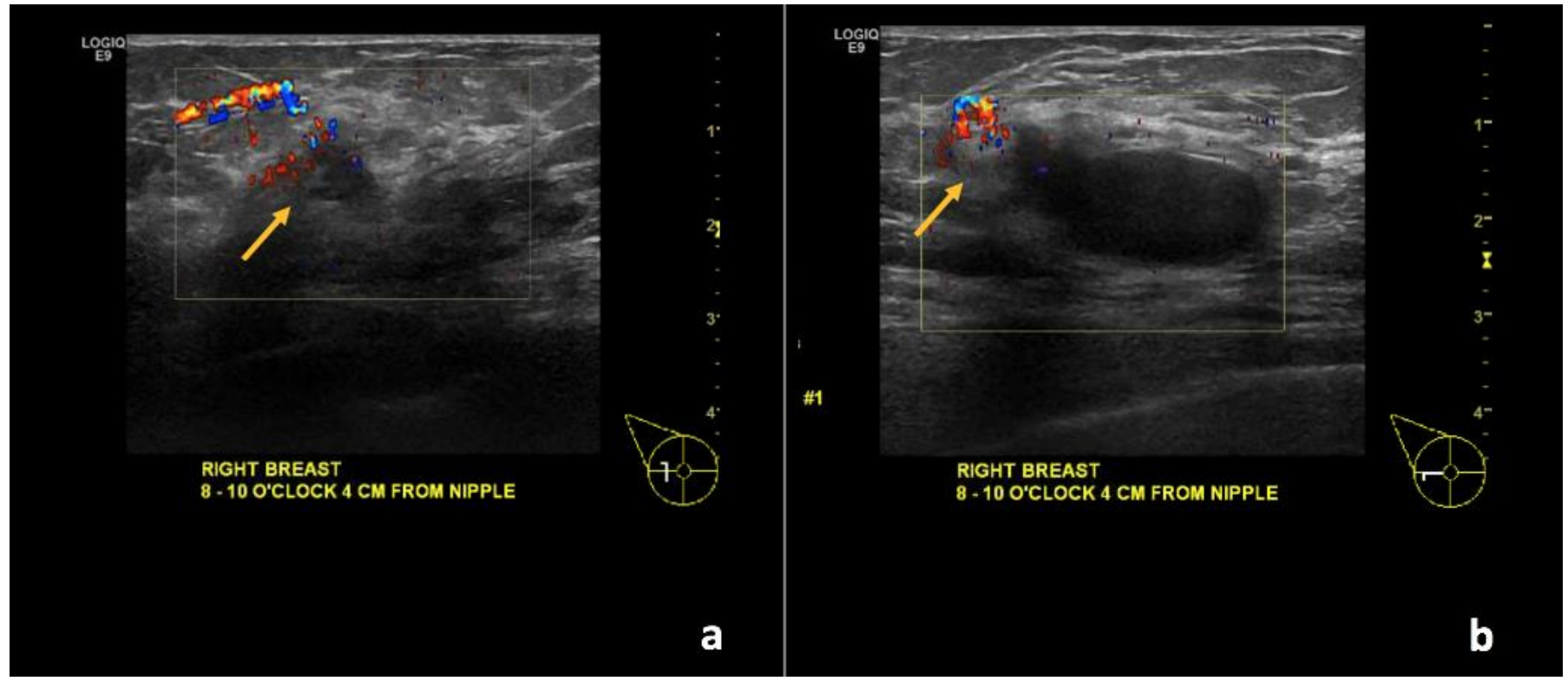

Figure 7: 67-year-old female with right breast invasive ductal carcinoma and ductal carcinoma in-situ.

Findings: (a,b) The known complex solid and cystic mass demonstrates interval decreased size of the cystic component with persistent, though less vascular, soft tissue component along the periphery (arrows).

Technique: Ultrasound and Color Doppler using a high frequency linear transducer (13 $\mathrm{MHz}$ ) after the completion of neoadjuvant chemotherapy.

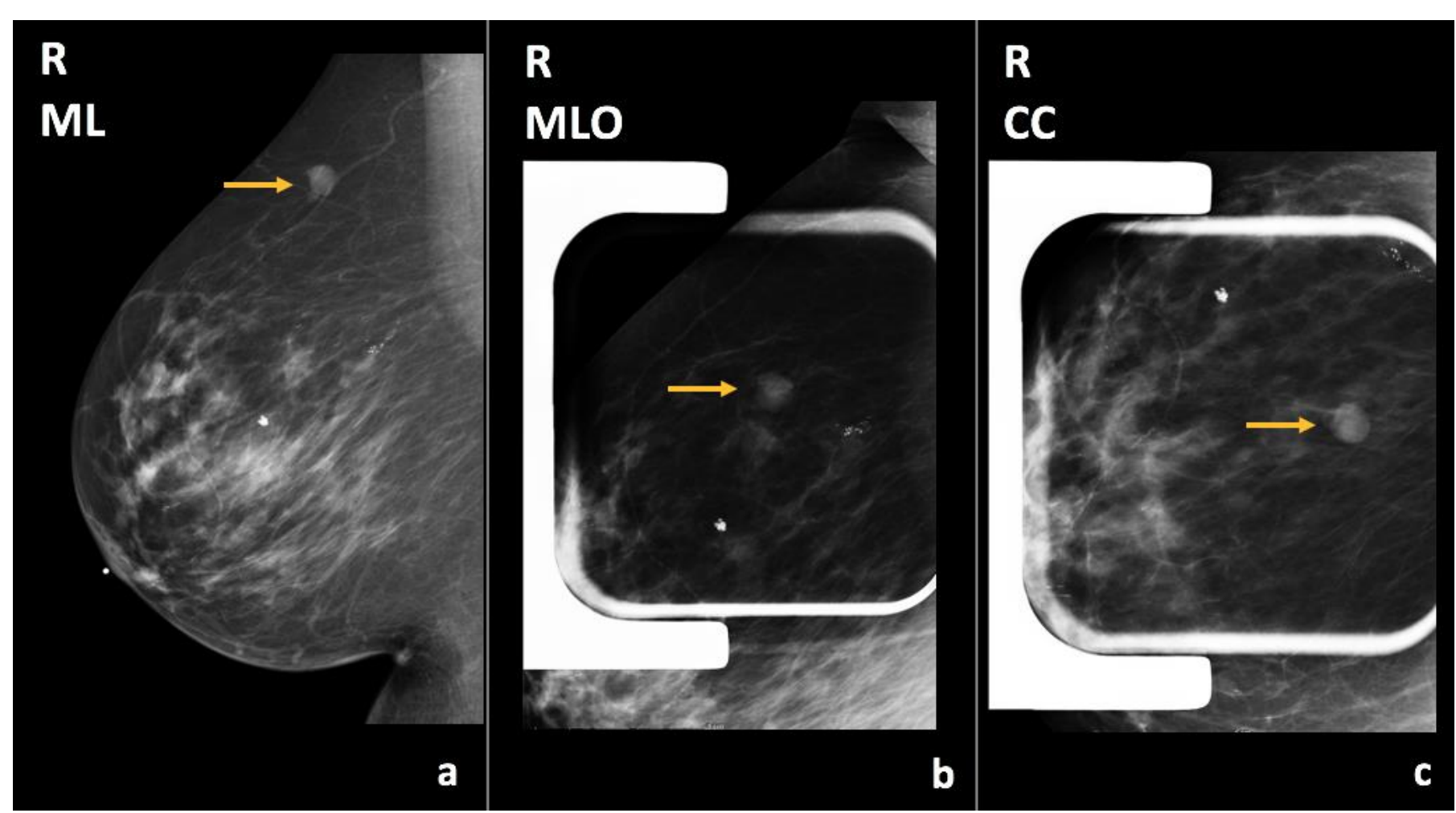

Figure 8: 73-year-old female with right breast infiltrating duct carcinoma. Two years prior to Figures 10 and 11.

Findings: There is a circumscribed mass in the upper central region of the right breast, posterior third depth (arrows).

Technique: Diagnostic mammogram (30 kVp, $64 \mathrm{mAs}$ ). (a) Mediolateral (ML), (b) mediolateral oblique (MLO) spot compression, and (c) craniocaudal (CC) spot compression views of the right breast. 


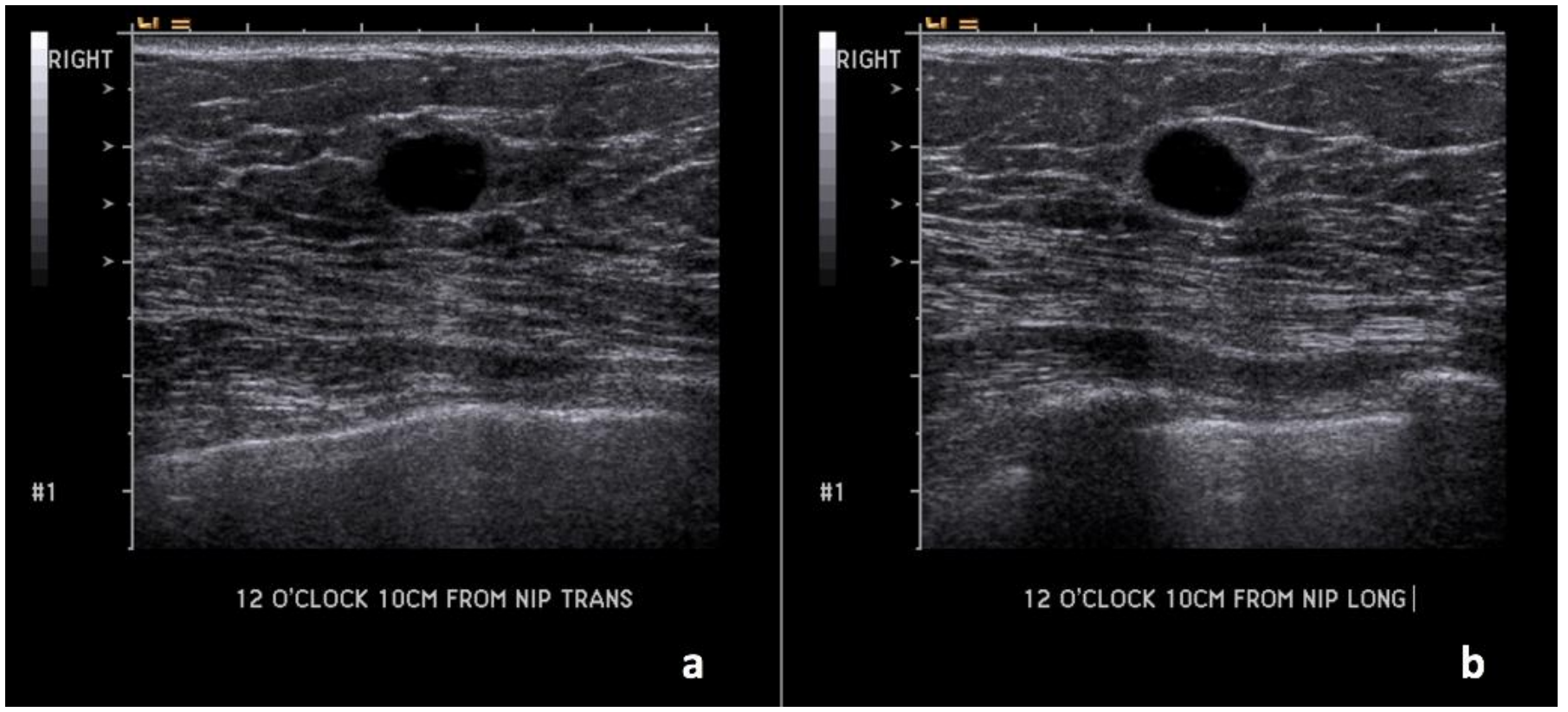

Figure 9: 73-year-old female with right breast infiltrating duct carcinoma. Two years prior to Figures 10 and 11.

Findings: (a, b) The right breast demonstrates an oval, circumscribed, parallel, anechoic mass with posterior acoustic enhancement at the 12 o'clock position, $10 \mathrm{~cm}$ from the nipple, measuring $1.0 \times 1.0 \times 0.8 \mathrm{~cm}$, corresponding to the mammographic abnormality, consistent with a simple cyst.

Technique: Ultrasound examination using a high frequency linear transducer (14 MHz).

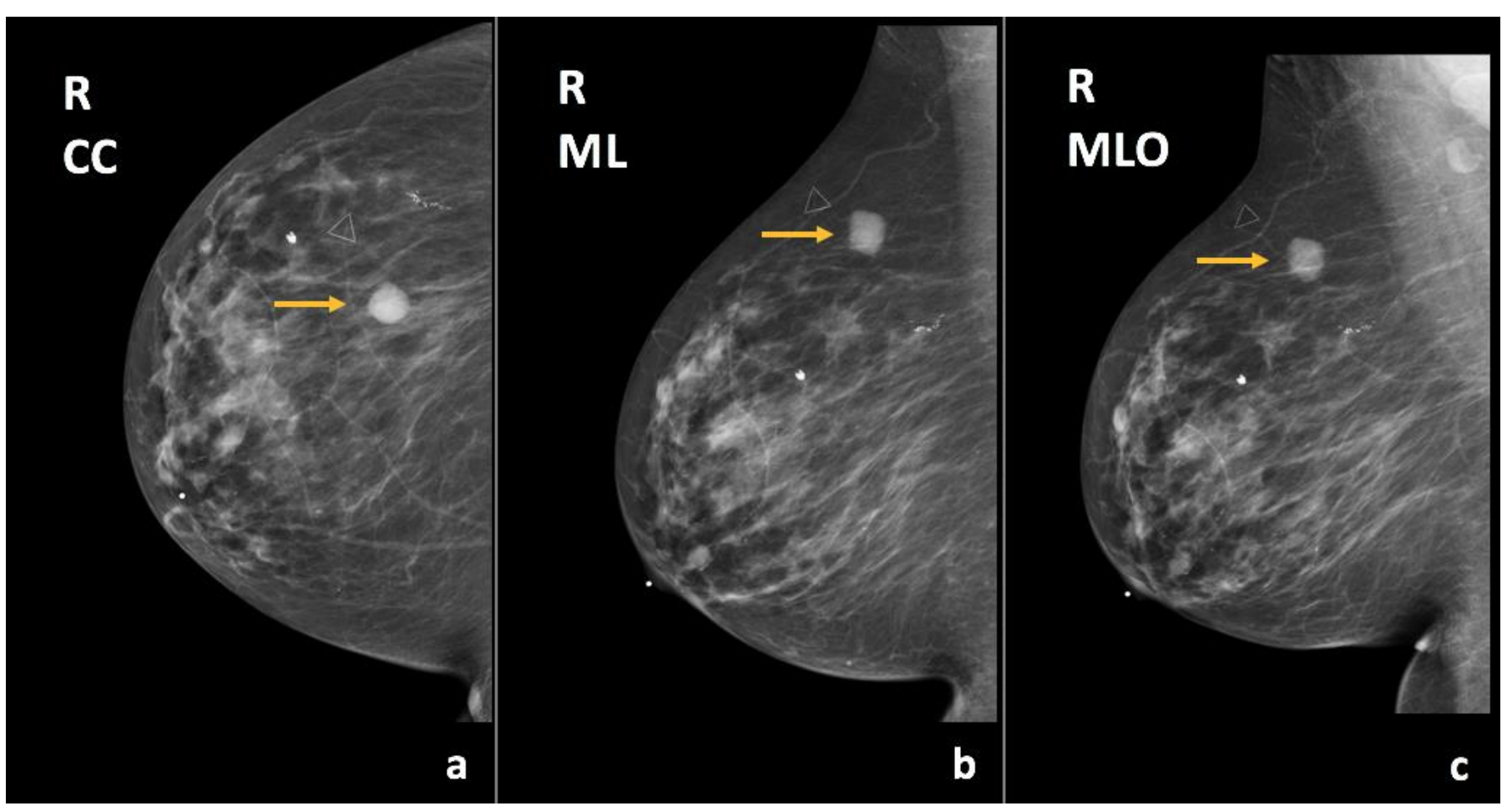

Figure 10: 73-year-old female with right breast infiltrating duct carcinoma.

Findings: There is an oval, circumscribed mass in the upper outer quadrant, posterior third depth (arrows), corresponding to known right breast cyst, which has slightly increased in size compared to the previous exam.

Technique: Diagnostic mammogram (29 kVp, $42 \mathrm{mAs}$ ). (a) Craniocaudal (CC), (b) mediolateral (ML), and (c) mediolateral oblique (MLO) views of the right breast. 


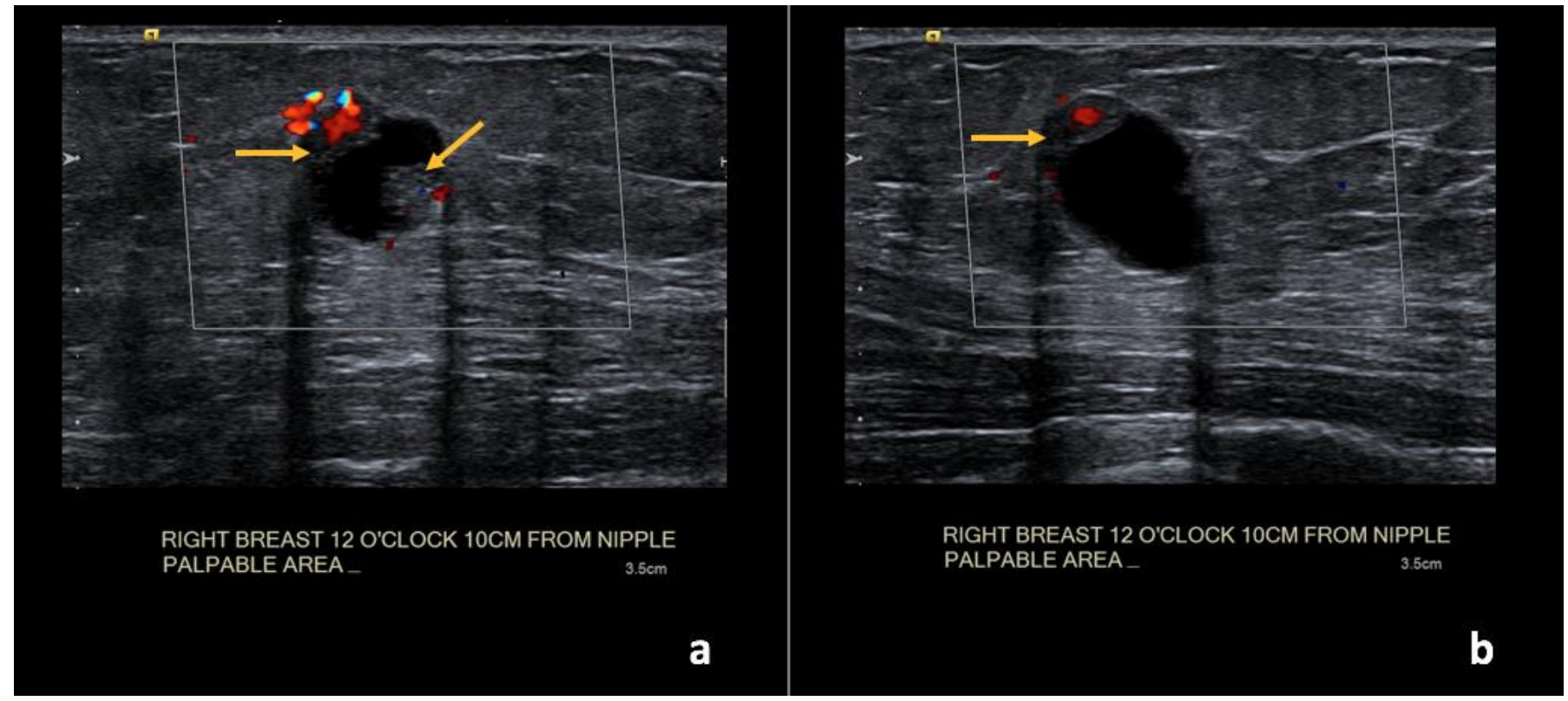

Figure 11: 73-year-old female with right breast infiltrating duct carcinoma.

Findings: (a, b) The right breast demonstrates an oval, circumscribed, complex solid and cystic mass at the 12 o'clock position, $10 \mathrm{~cm}$ from the nipple, measuring $1.6 \times 1.1 \times 1.2 \mathrm{~cm}$ with new vascular internal mural nodularity (arrows).

Technique: Ultrasound and Color Doppler examination using a high frequency linear transducer (12 MHz).

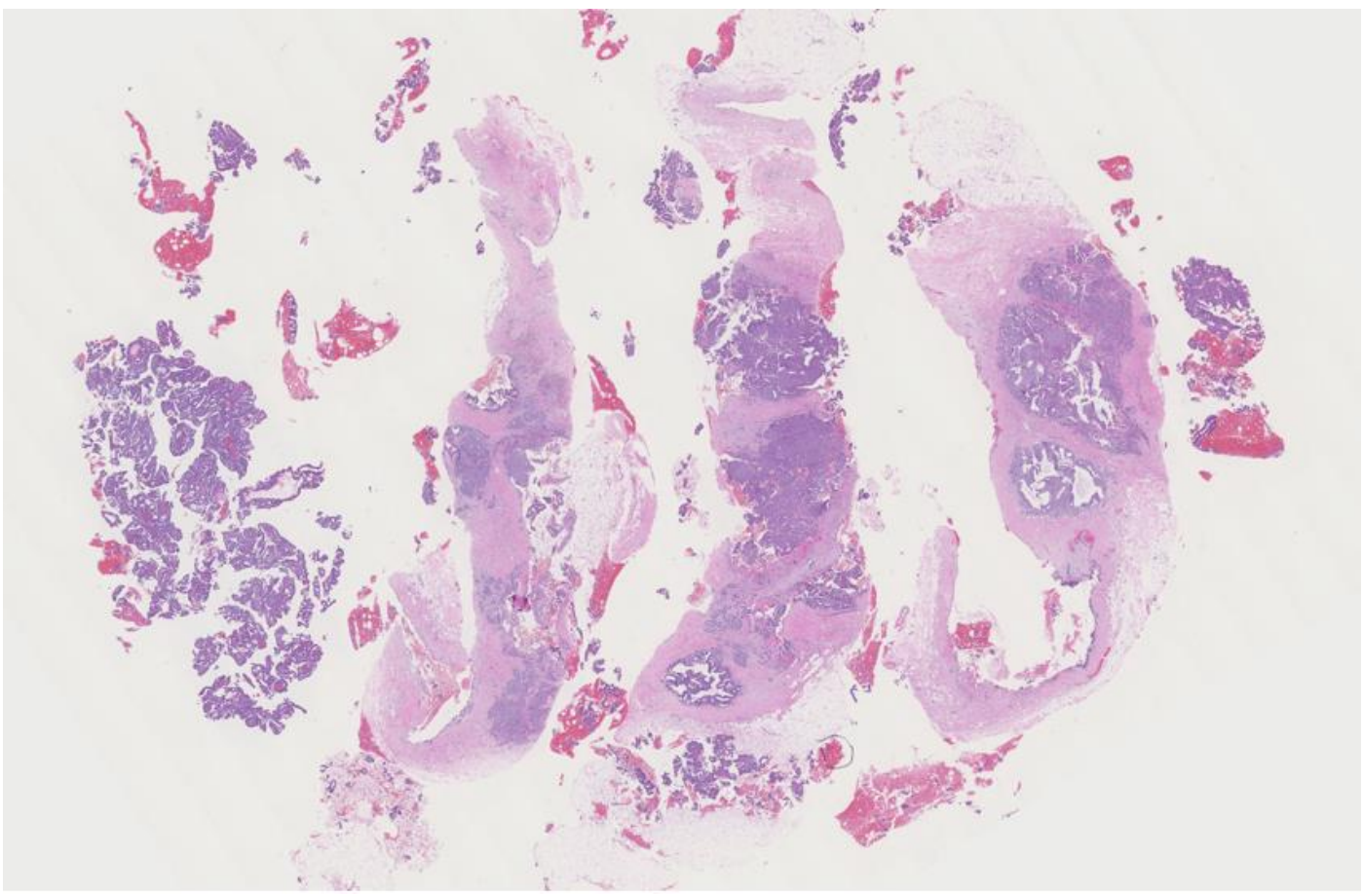

Figure 12: 73-year-old female with right breast infiltrating duct carcinoma.

Findings: There is extensive intra-cystic ductal carcinoma in-situ, cribriform and papillary types, Van Nuys grade 1 of 3 and infiltrating well differentiated duct carcinoma, grade 1 of 3, Nottingham score of 4.

Technique: Microscopic examination using H\&E stain. Low-power view $(7 \mathrm{x})$ from the core needle biopsy of a right breast complex solid and cystic mass. 


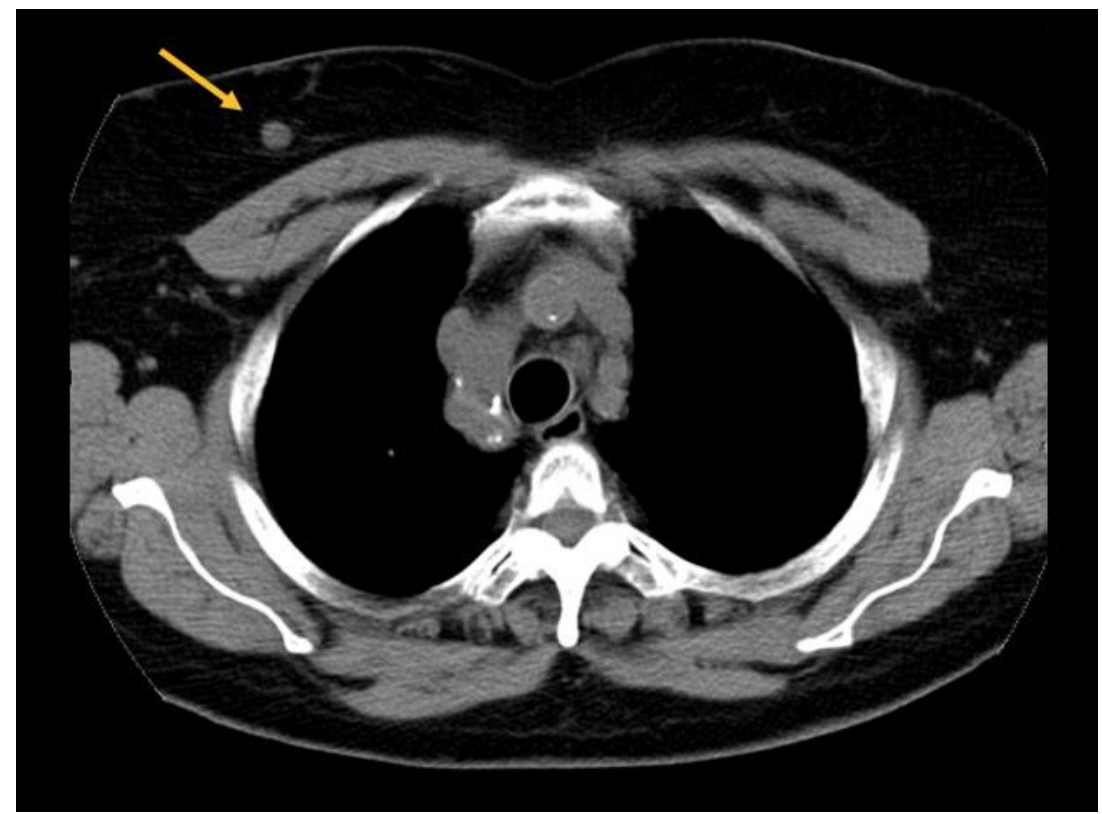

Figure 13: 76-year-old female with right breast infiltrating differentiated duct carcinoma with mucinous features.

Findings: Incidentally identified $0.9 \mathrm{~cm}$ nodule in the right breast (arrow).

Technique: Non-contrast enhanced computed tomography (CT) of the chest. Axial plane through the level of the upper chest. (GE Medical Systems, Brightspeed; $120 \mathrm{kVp}, 434 \mathrm{~mA}, 5.0 \mathrm{~mm}$ slice thickness).

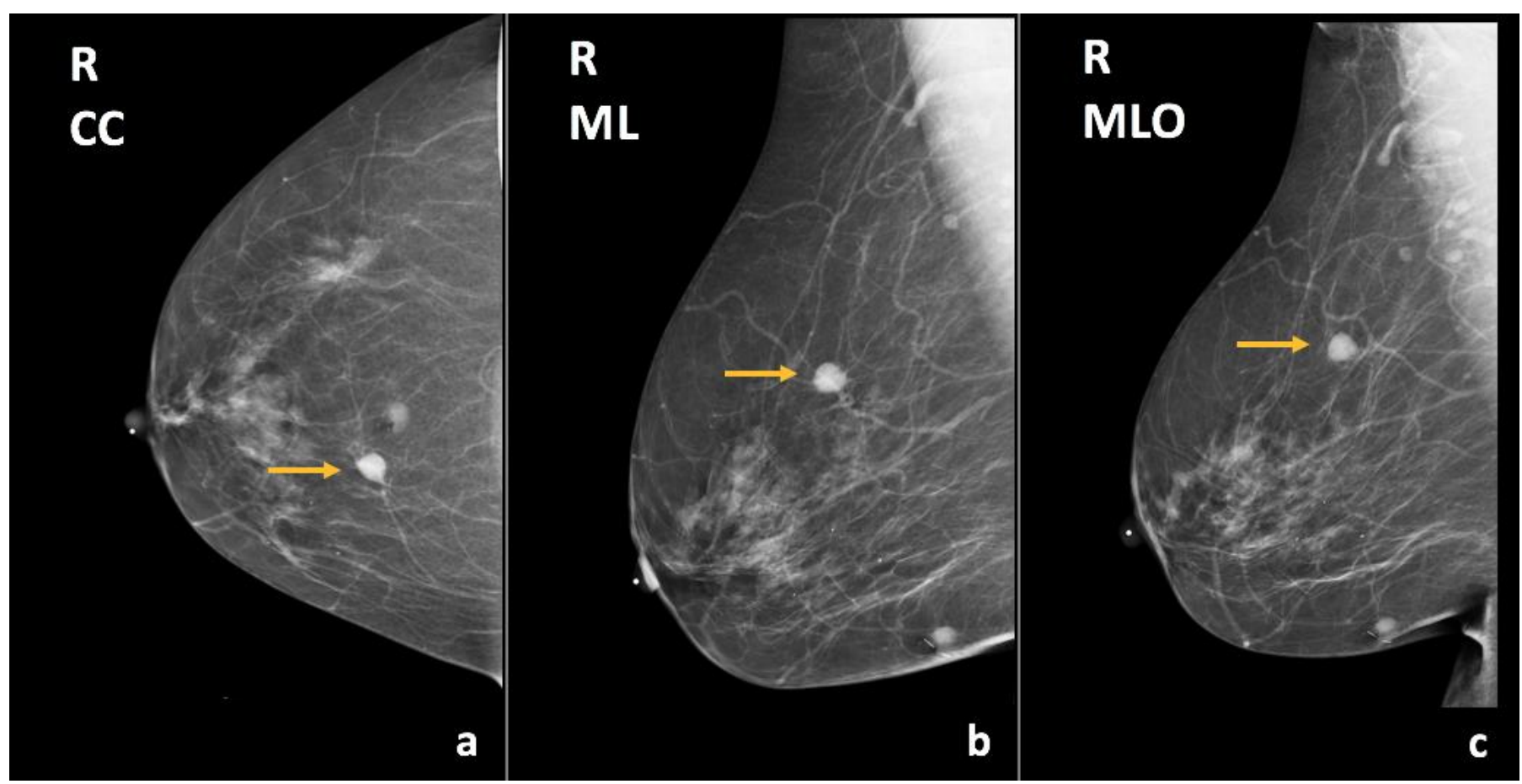

Figure 14: 76-year-old female with right breast infiltrating differentiated duct carcinoma with mucinous features.

Findings: There is a hyperdense, round, circumscribed mass in the upper inner quadrant, middle third depth (arrows), correlating with the finding on prior CT (figure 13).

Technique: Diagnostic mammogram (29 kVp, $44 \mathrm{mAs}$ ). (a) Craniocaudal (CC), (b) mediolateral (ML), and (c) mediolateral oblique (MLO) views of the right breast. 


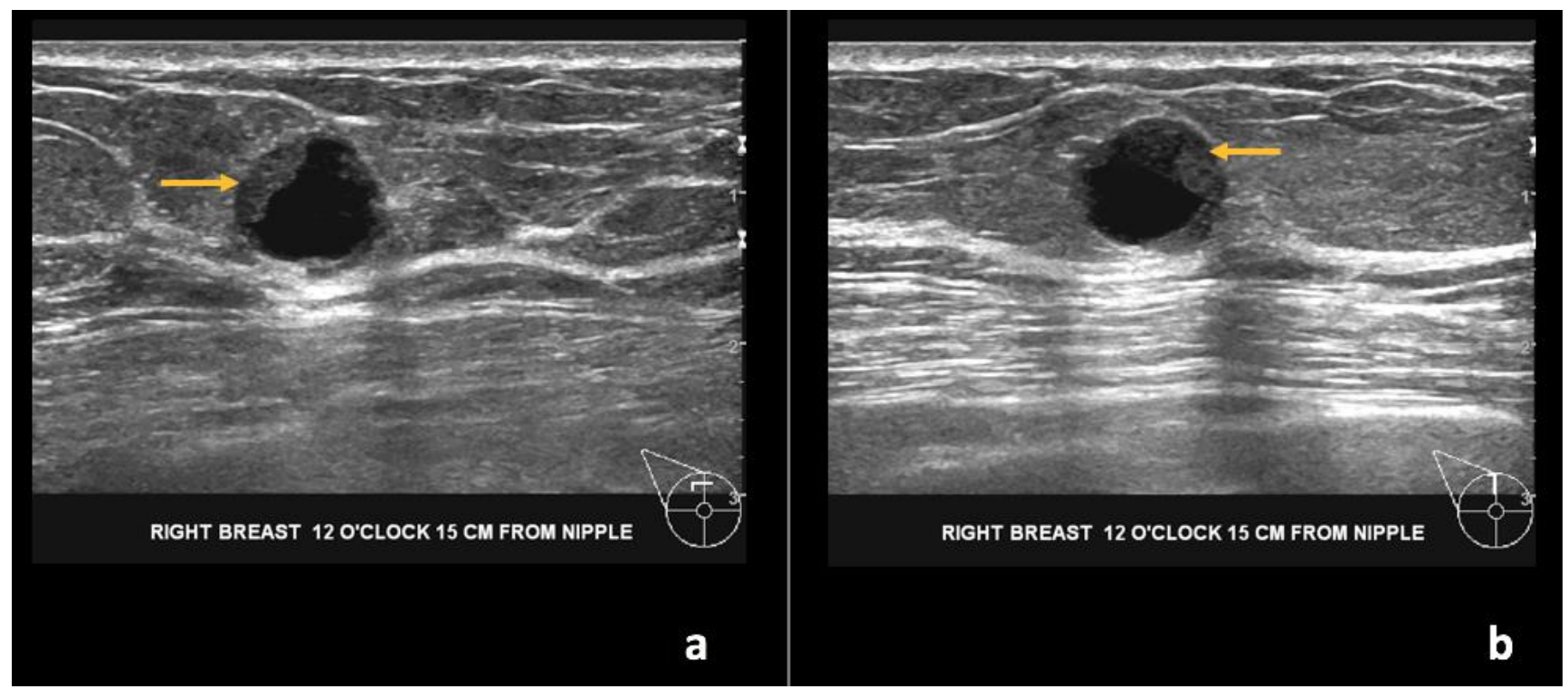

Figure 15: 76-year-old female with right breast infiltrating differentiated duct carcinoma with mucinous features.

Findings: (a, b) The right breast demonstrates a round, circumscribed, complex solid and cystic mass at the 12 o'clock position, $15 \mathrm{~cm}$ from the nipple measuring $1.1 \times 0.9 \times 1.1 \mathrm{~cm}$ (arrows at solid component).

Technique: Ultrasound examination using a high frequency linear transducer (13 MHz).

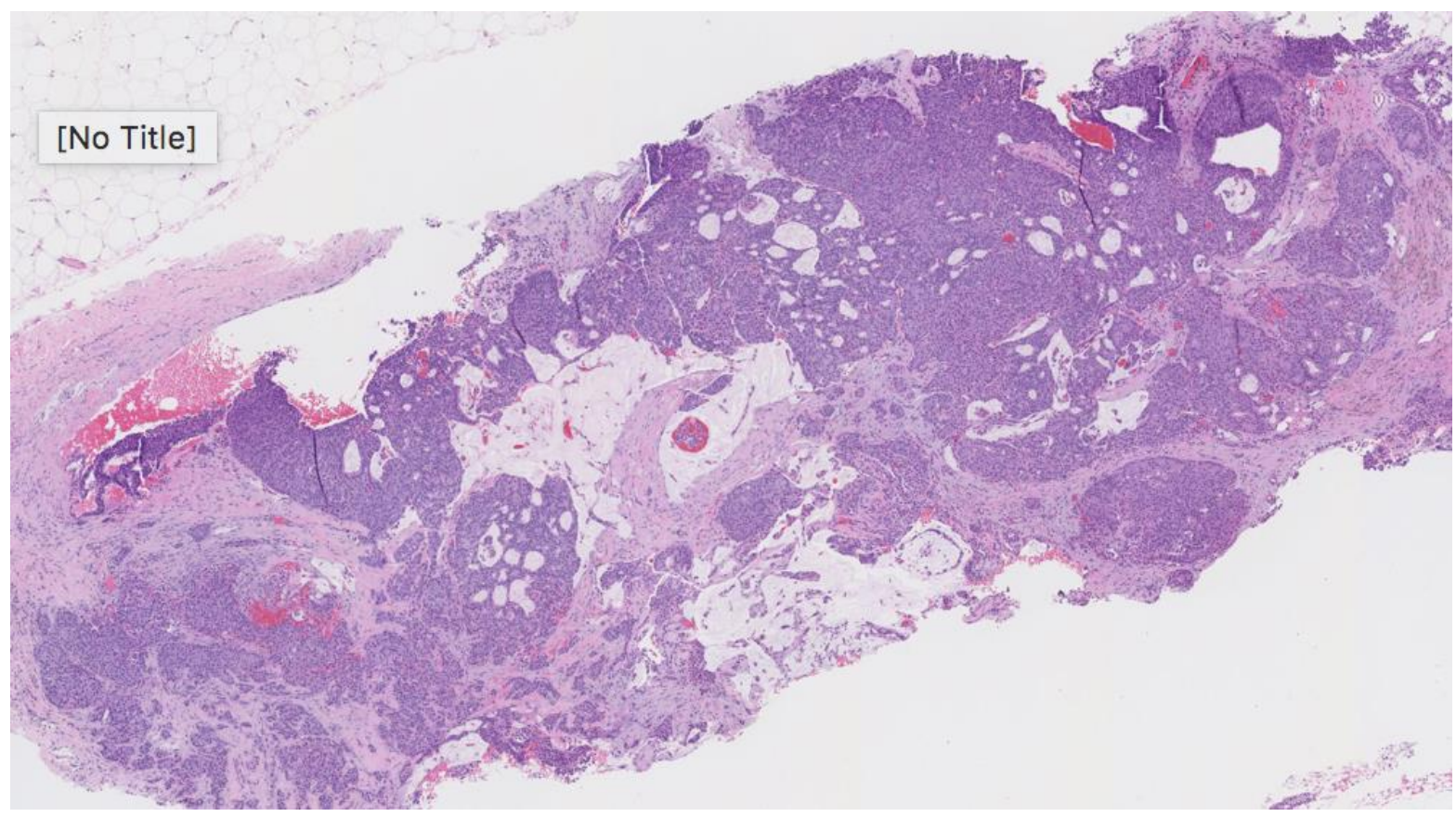

Figure 16: 76-year-old female with right breast infiltrating differentiated duct carcinoma with mucinous features.

Findings: There is moderately differentiated invasive ductal carcinoma, grade 2 of 3, Nottingham score of 7. Mucin lakes are noticed in the center of the tissue obtained.

Technique: Microscopic examination using H\&E stain. Medium-power view (35x) from the core needle biopsy of the right breast complex solid and cystic mass. 


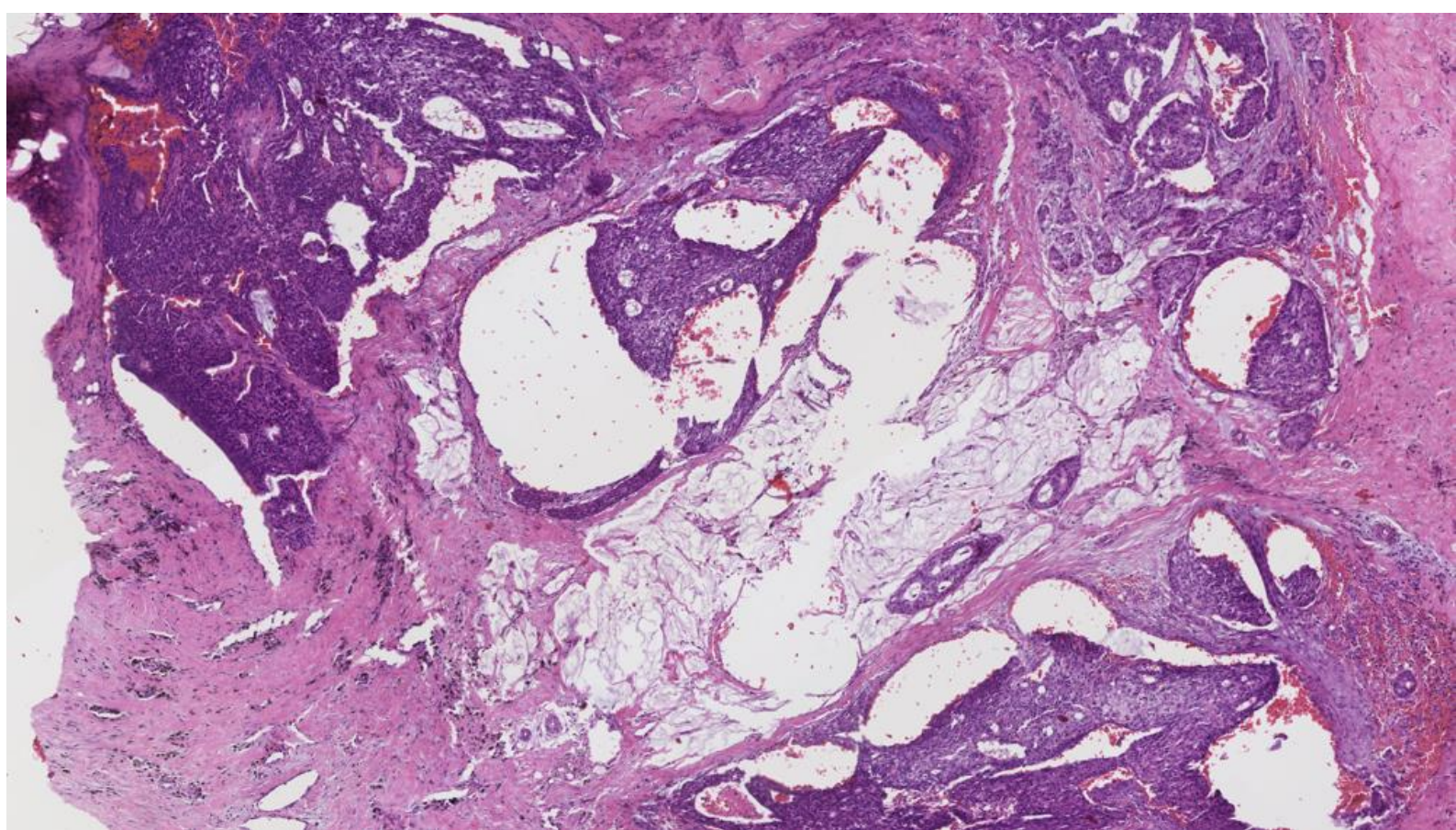

Figure 17: 76-year-old female with right breast infiltrating differentiated duct carcinoma with mucinous features.

Findings: There is moderately differentiated invasive ductal carcinoma, grade 2 of 3 , Nottingham score of 7 . Mucin lakes are noticed in the center of the tissue obtained from partial right breast mastectomy, showing a similar lesion seen in the core needle biopsy (Figure 16).
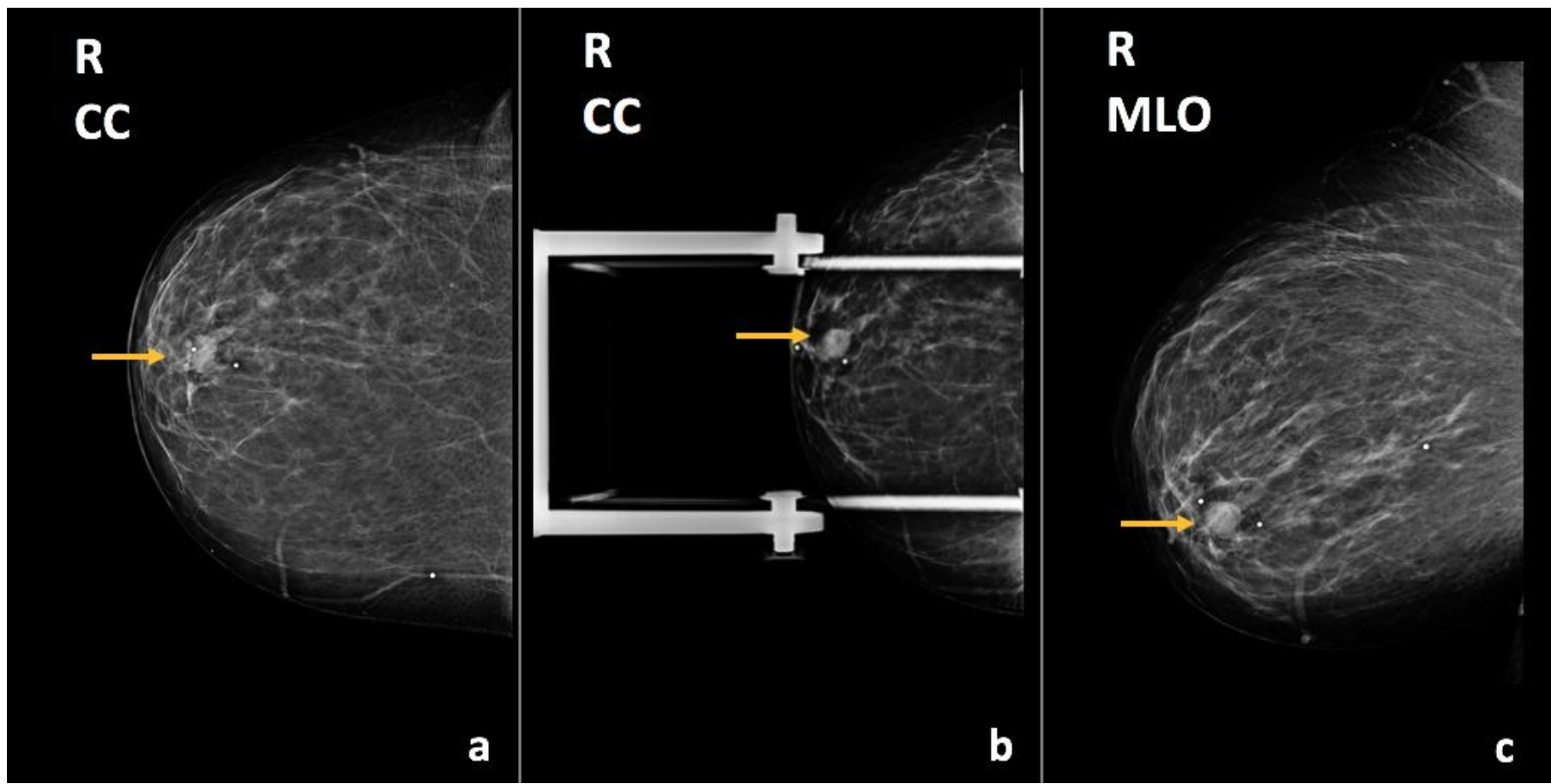

Figure 18: 52-year-old female with right breast ductal carcinoma in-situ.

Findings: There is an oval, circumscribed mass in the retroareolar region (arrows).

Technique: Diagnostic mammogram, outside hospital (kVp and mAs unavailable). (a) Craniocaudal (CC), (b) craniocaudal (CC) spot compression, and (c) mediolateral oblique views of the right breast. 


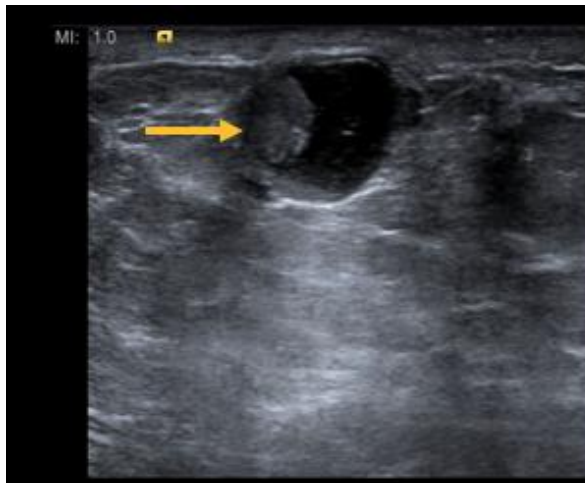

RT Breast 6:00 Nipple PALPLE AREA TRANS a

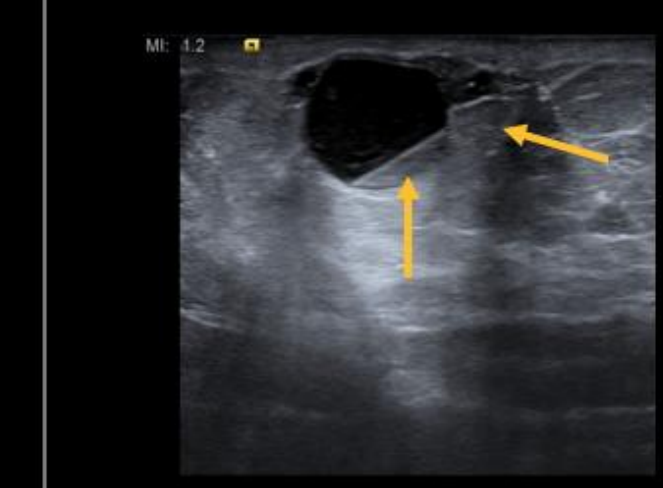

RT Breast 6:00 Nipple PALPLE AREA LONG

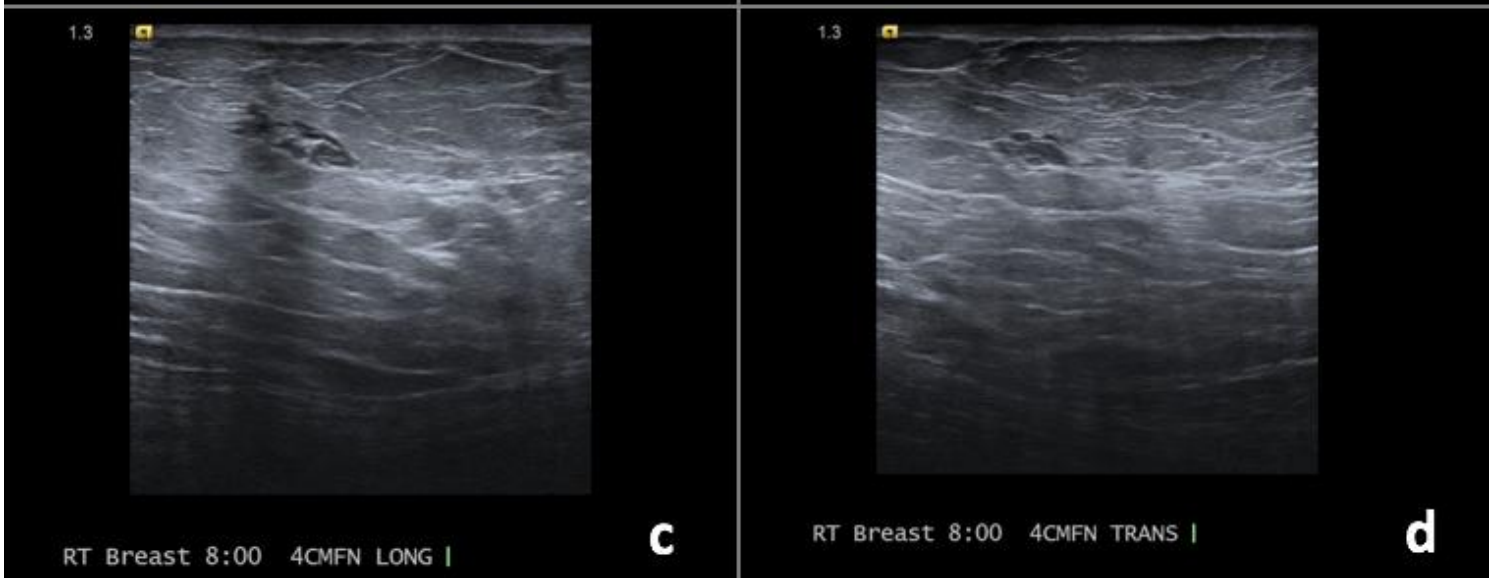

Figure 19: 52-year-old female with right breast ductal carcinoma in-situ.

Findings: (a, b) The right breast demonstrates a round, circumscribed, complex solid and cystic mass (arrows at solid component) at the 6 o'clock axis corresponding to the palpable abnormality. (c, d) Additionally within the right breast, there is a complex solid and cystic mass at the 8 o'clock position, $4 \mathrm{~cm}$ from the nipple.

Technique: Ultrasound examination using a high frequency linear transducer (15 MHz).

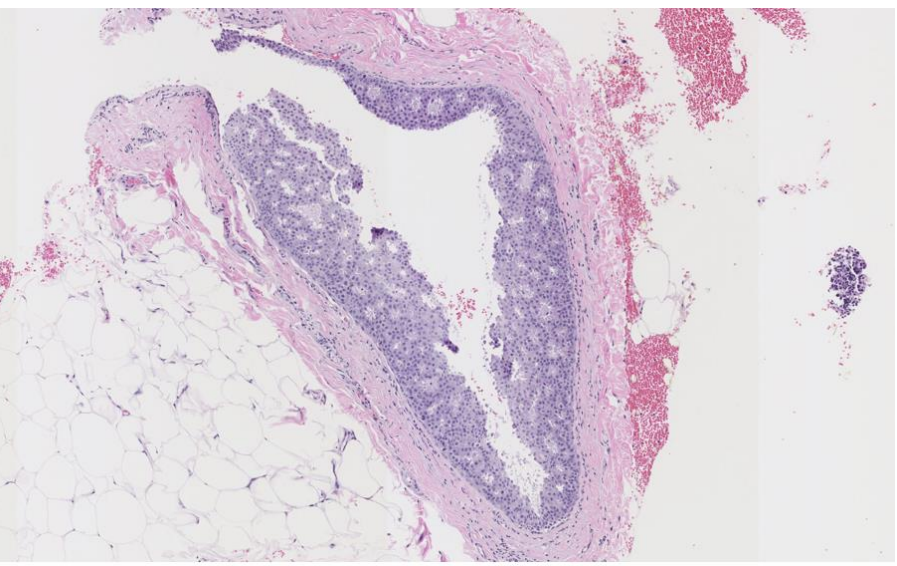

Figure 20 (left): 52-year-old female with right breast ductal carcinoma in-situ.

Findings: There is cribriform type of ductal carcinoma in-situ, Van Nuys grade 1 of 3.

Technique: Microscopic examination using H\&E stain. Medium-power view (75x) from the core needle biopsy of a right breast complex solid and cystic mass. 


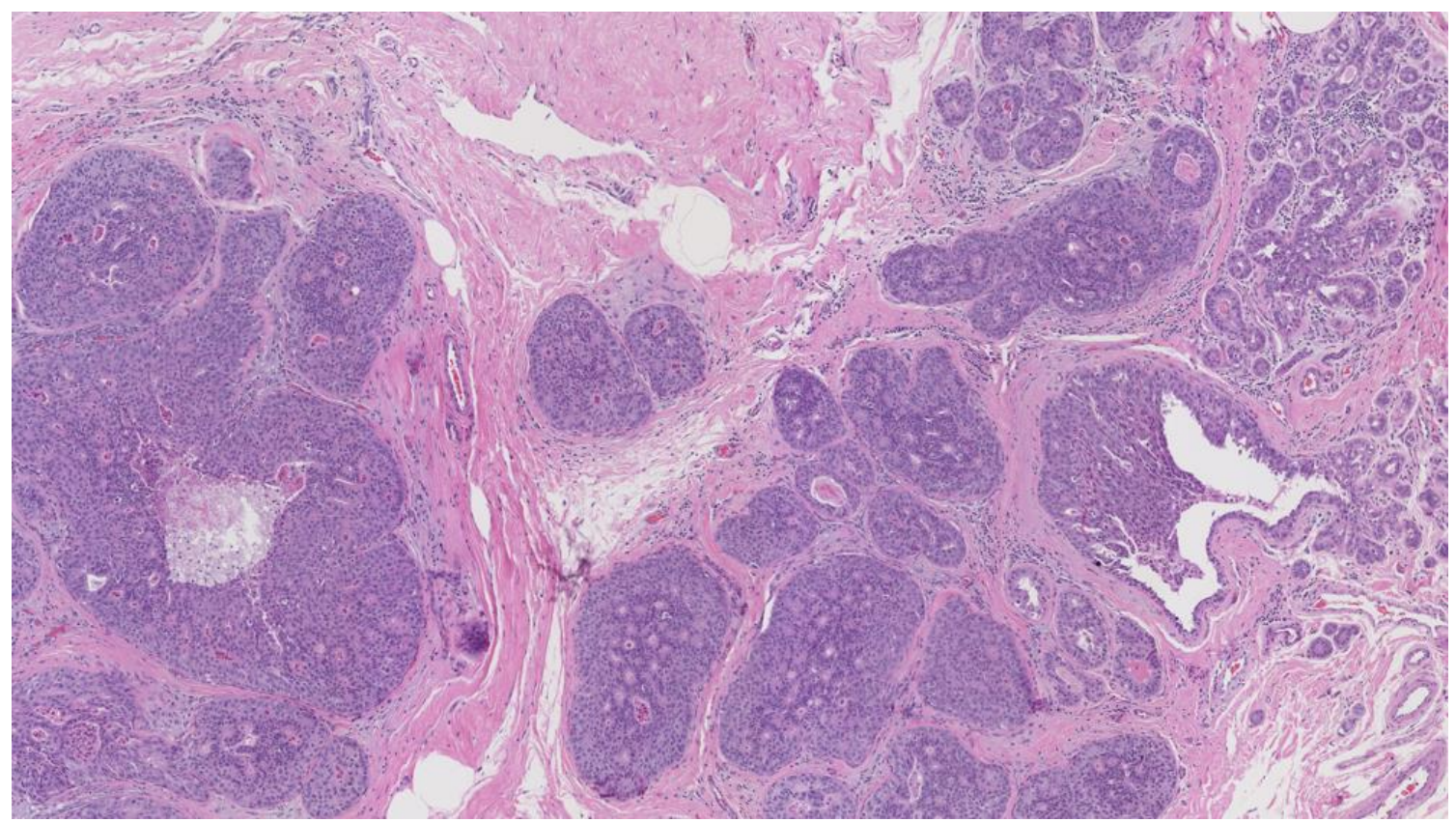

Figure 21: 52-year-old female with right breast ductal carcinoma in-situ.

Findings: There is cribriform type of ductal carcinoma in-situ, Van Nuys grade 2 of 3.

Technique: Microscopic examination using H\&E stain. Medium-power view (55x) from the total right breast mastectomy.

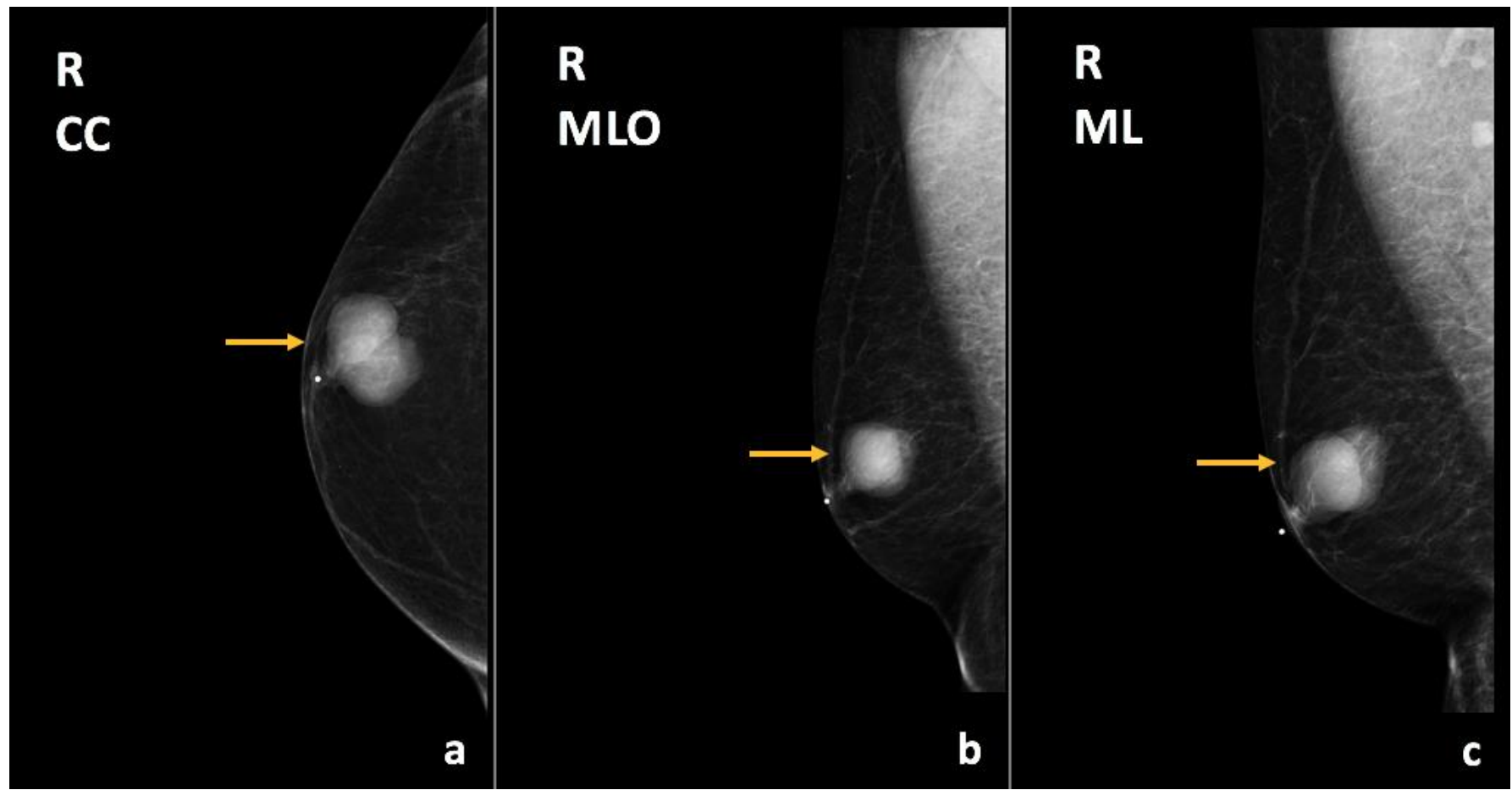

Figure 22: 62-year-old male with right breast encapsulated papillary carcinoma.

Findings: There is an oval, circumscribed, hyperdense mass in the retroareolar region (arrows), corresponding to the palpable abnormality.

Technique: Diagnostic mammogram (34 kVp, $42 \mathrm{mAs}$ ). (a) Craniocaudal (CC), (b) mediolateral oblique (MLO), and (c) mediolateral (ML) views of the right breast. 


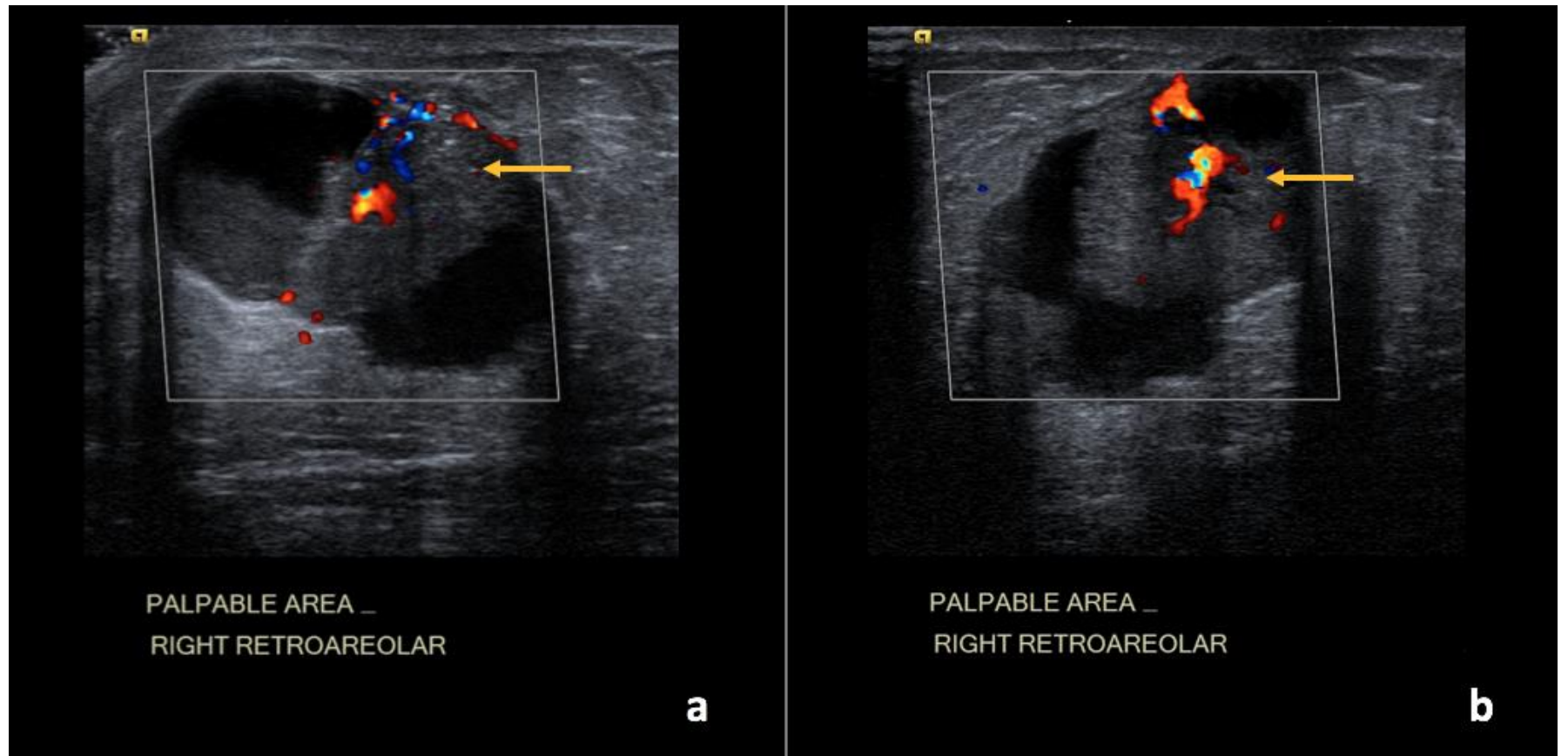

Figure 23: 62-year-old male with right breast encapsulated papillary carcinoma.

Findings: (a, b) The right breast demonstrates an irregular complex solid and cystic mass in the retroareolar region measuring 3.5 x $3.1 \times 2.9 \mathrm{~cm}$ with increased vascularity within the solid components of the mass (arrows).

Technique: Ultrasound and Color Doppler examination using a high frequency linear transducer (12 MHz).

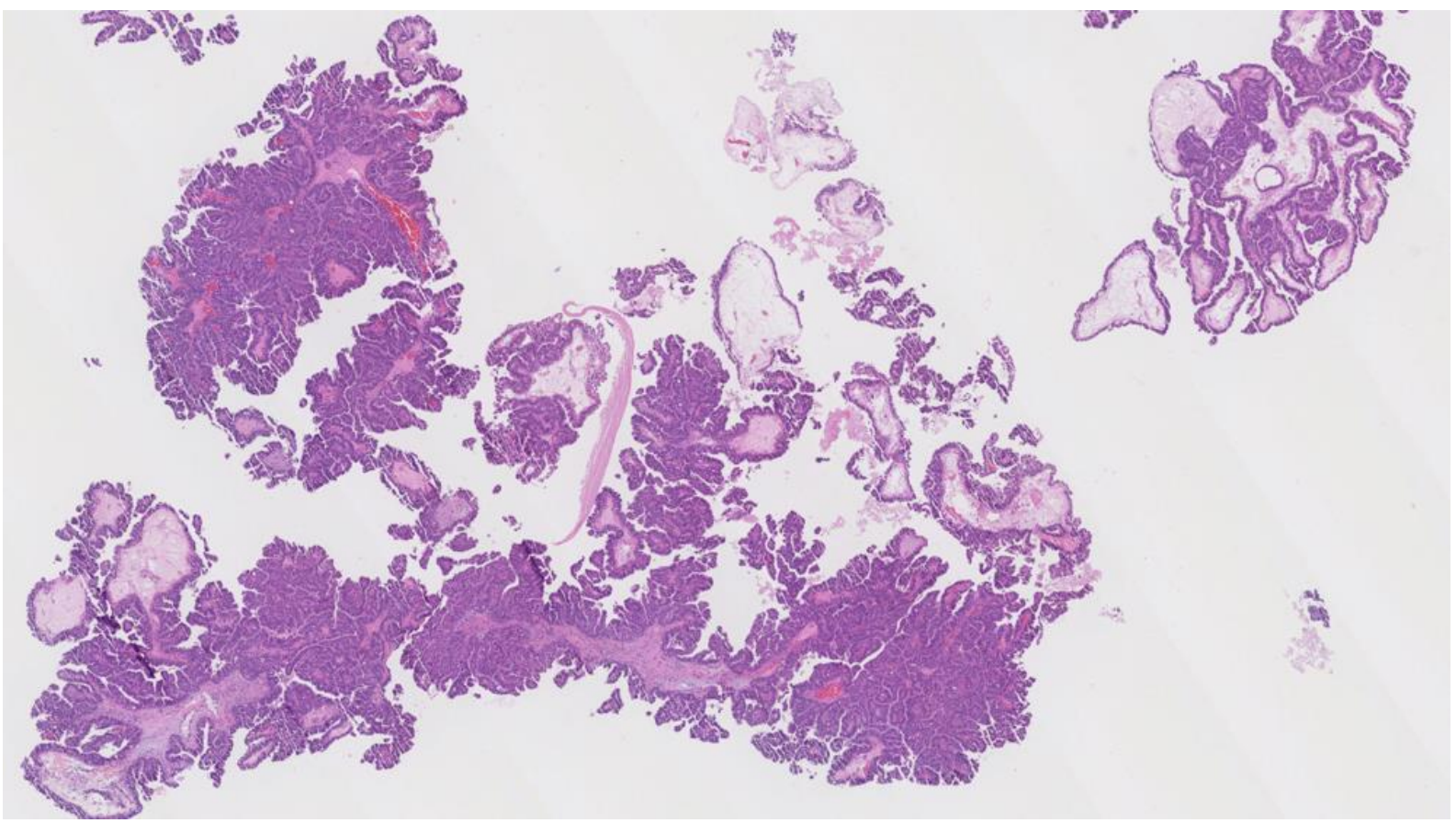

Figure 24: 62-year-old male with right breast encapsulated papillary carcinoma.

Findings: There is solid and papillary types of ductal carcinoma in-situ, Van Nuys grade 2 of 3 , with atypical papillary proliferation and fibrous connective tissue and vascular cores.

Technique: Microscopic examination using H\&E stain. Medium-power view (20x) from the core needle biopsy of a right breast complex solid and cystic mass. 


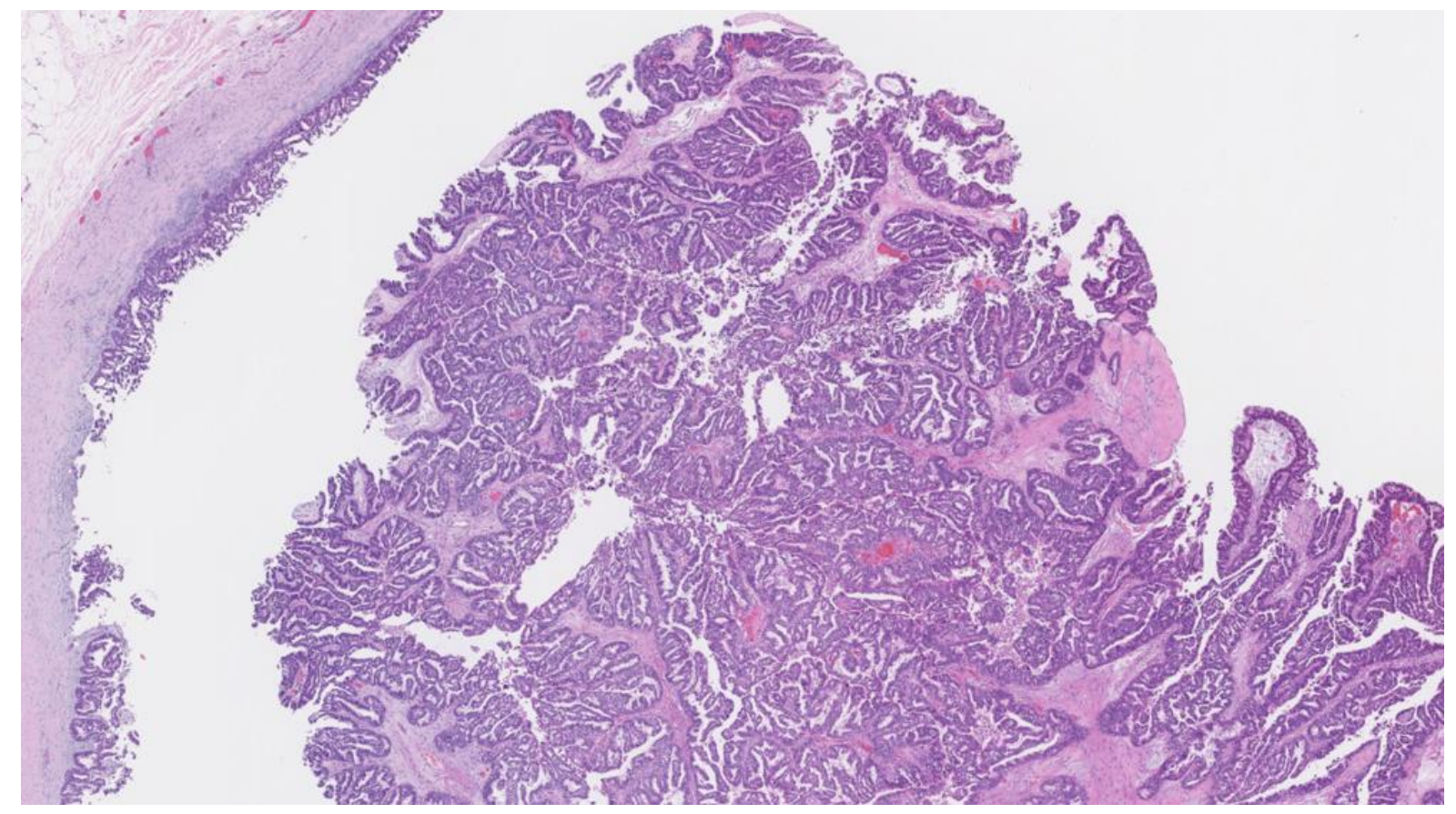

Figure 25: 62-year-old male with right breast encapsulated papillary carcinoma.

Findings: There is extensive intra-cystic papillary carcinoma encased by a thick fibrotic capsule.

Technique: Microscopic examination. Medium-power view (50x) from the total right breast mastectomy.

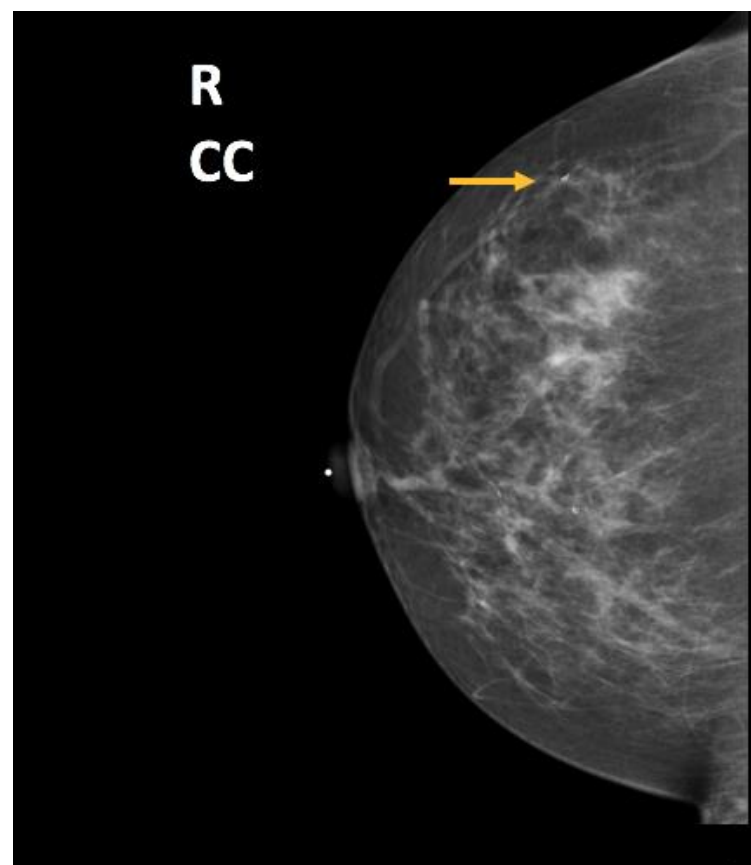

\section{$\mathbf{R}$ \\ MLO}

a

Figure 26: 47-year-old female with right breast metaplastic carcinoma. Seven months prior to Figures 27 and 28.

Findings: There is a biopsy clip (arrows) in the upper outer quadrant with several benign calcifications and no suspicious findings when compared with prior studies.

Technique: Screening mammogram (31 kVp, $64 \mathrm{mAs})$. (a) Craniocaudal (CC) and (b) mediolateral oblique (MLO) views of the right breast. 


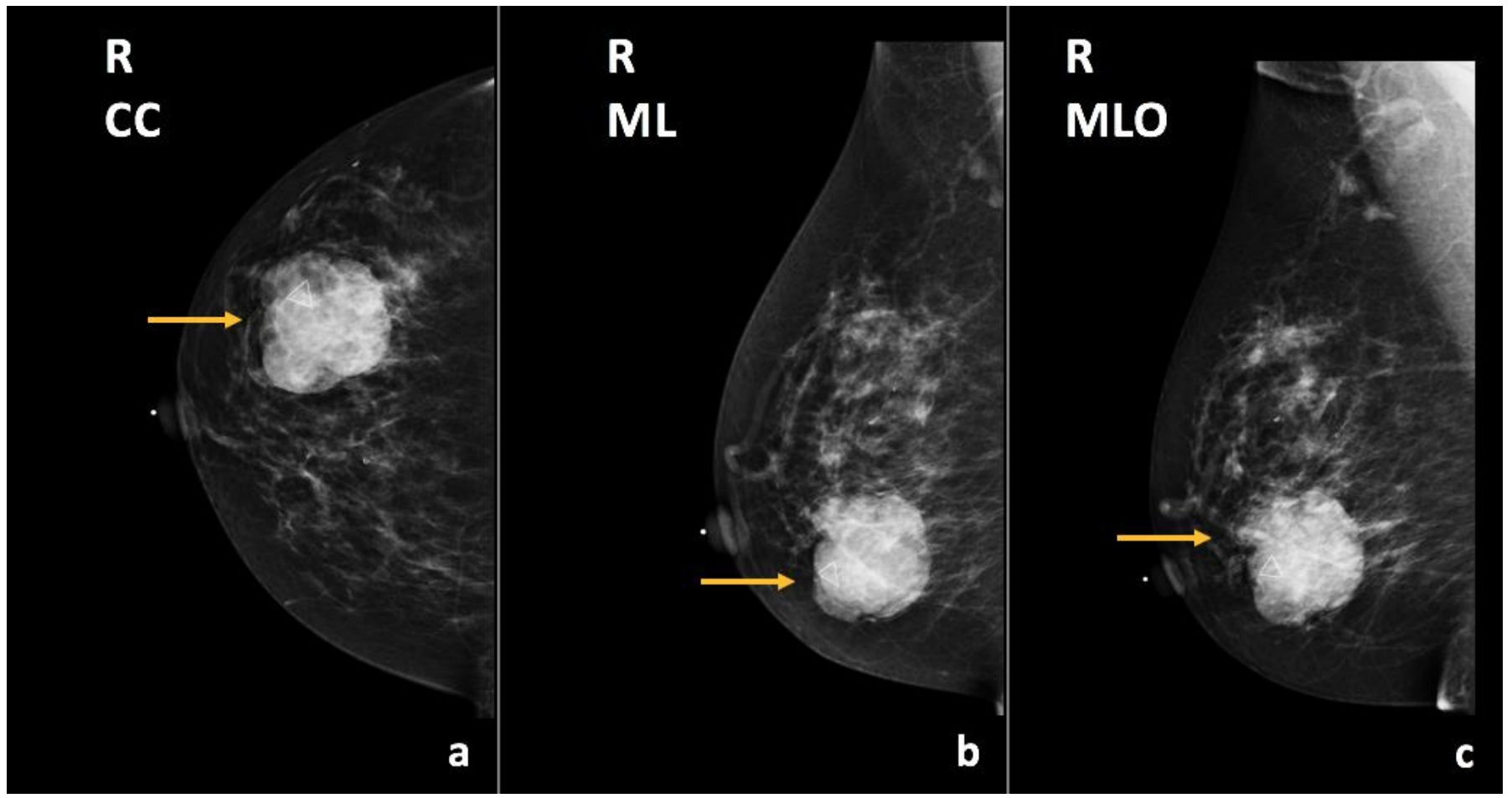

Figure 27: 47-year-old female with right breast metaplastic carcinoma.

Findings: There is a circumscribed, round mass in the lower outer quadrant of the right breast, anterior and middle third depth (arrows), corresponding to the palpable abnormality.

Technique: Diagnostic mammogram (30 kV, $94 \mathrm{mAs}$ ). (a) Craniocaudal (CC), (b) mediolateral (ML), and (c) mediolateral oblique (MLO) views of the right breast.

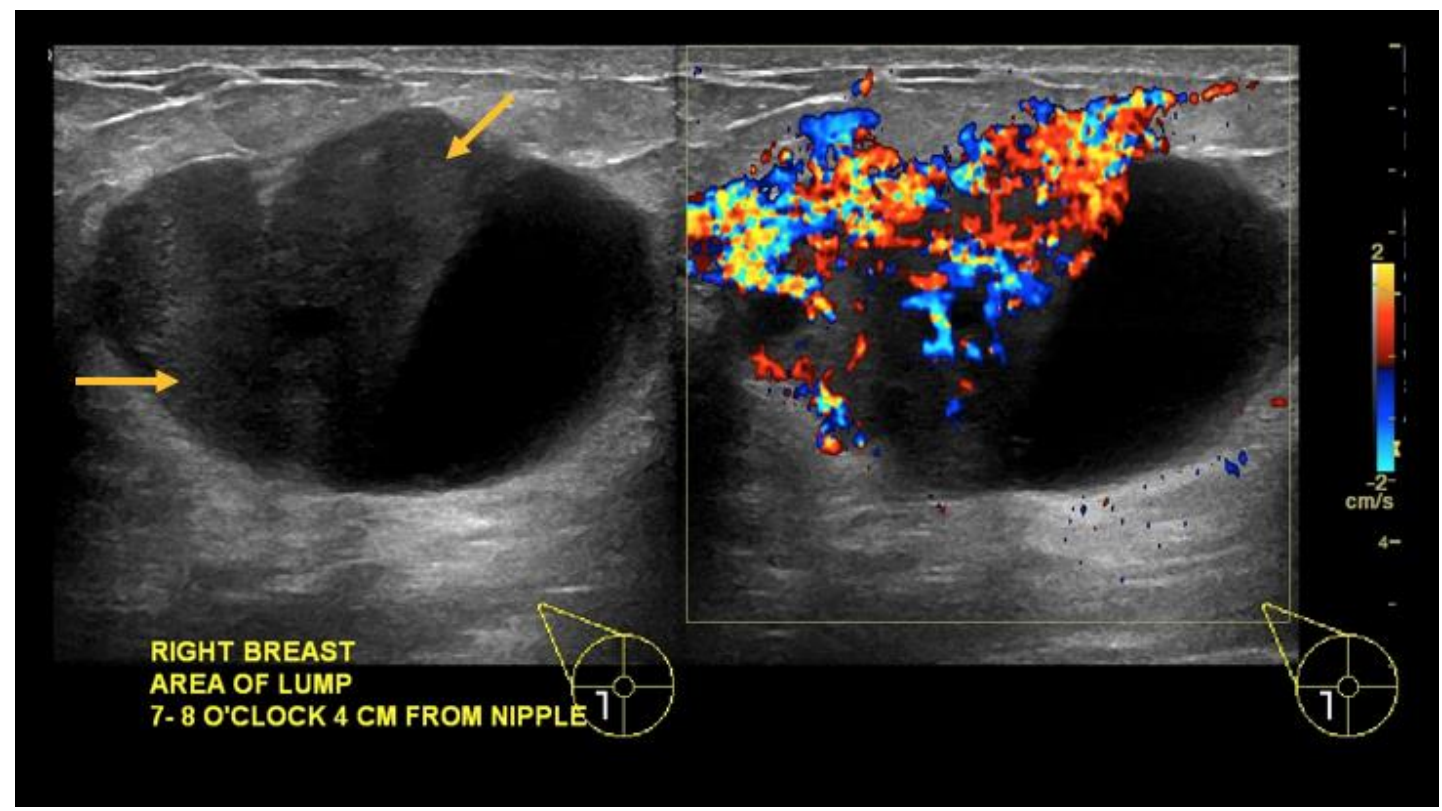

Figure 28: 47-year-old female with right breast metaplastic carcinoma.

Findings: (a, b) The right breast demonstrates an oval, parallel, complex solid and cystic mass between the 7 o'clock to 8 o'clock position, $4 \mathrm{~cm}$ from the nipple, measuring $4.9 \times 3.1 \times 4.9 \mathrm{~cm}$, with focally indistinct margins and increased vascularity of the solid components (arrows).

Technique: Ultrasound and Color Doppler examination using a high frequency linear transducer (13 MHz). 


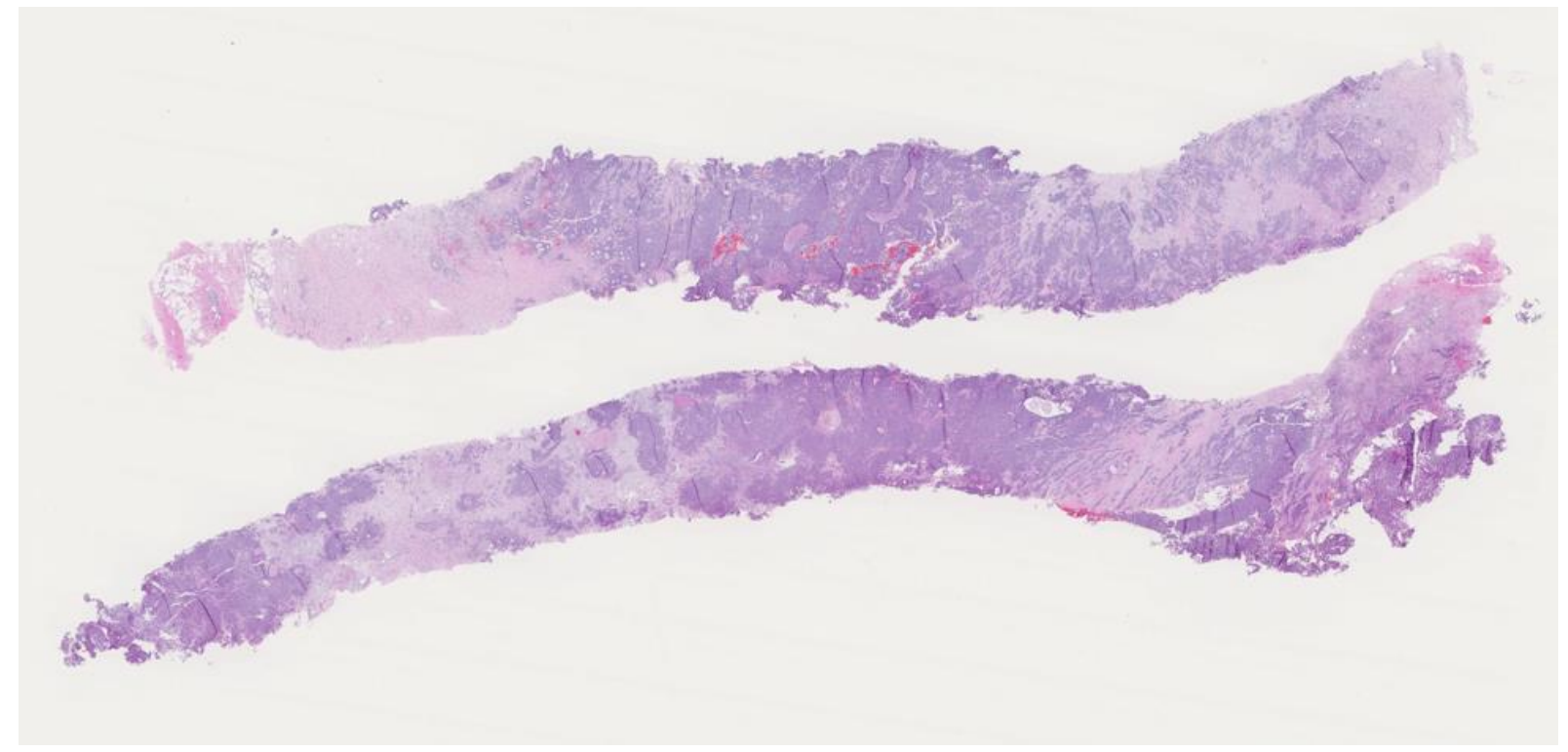

Figure 29: 47-year-old female with right breast metaplastic carcinoma.

Findings: There is predominantly solid, infiltrating mammary carcinoma, Nottingham score of 7.

Technique: Microscopic examination using H\&E stain. Low-power view (8x) from the core needle biopsy of a right breast complex solid and cystic mass.

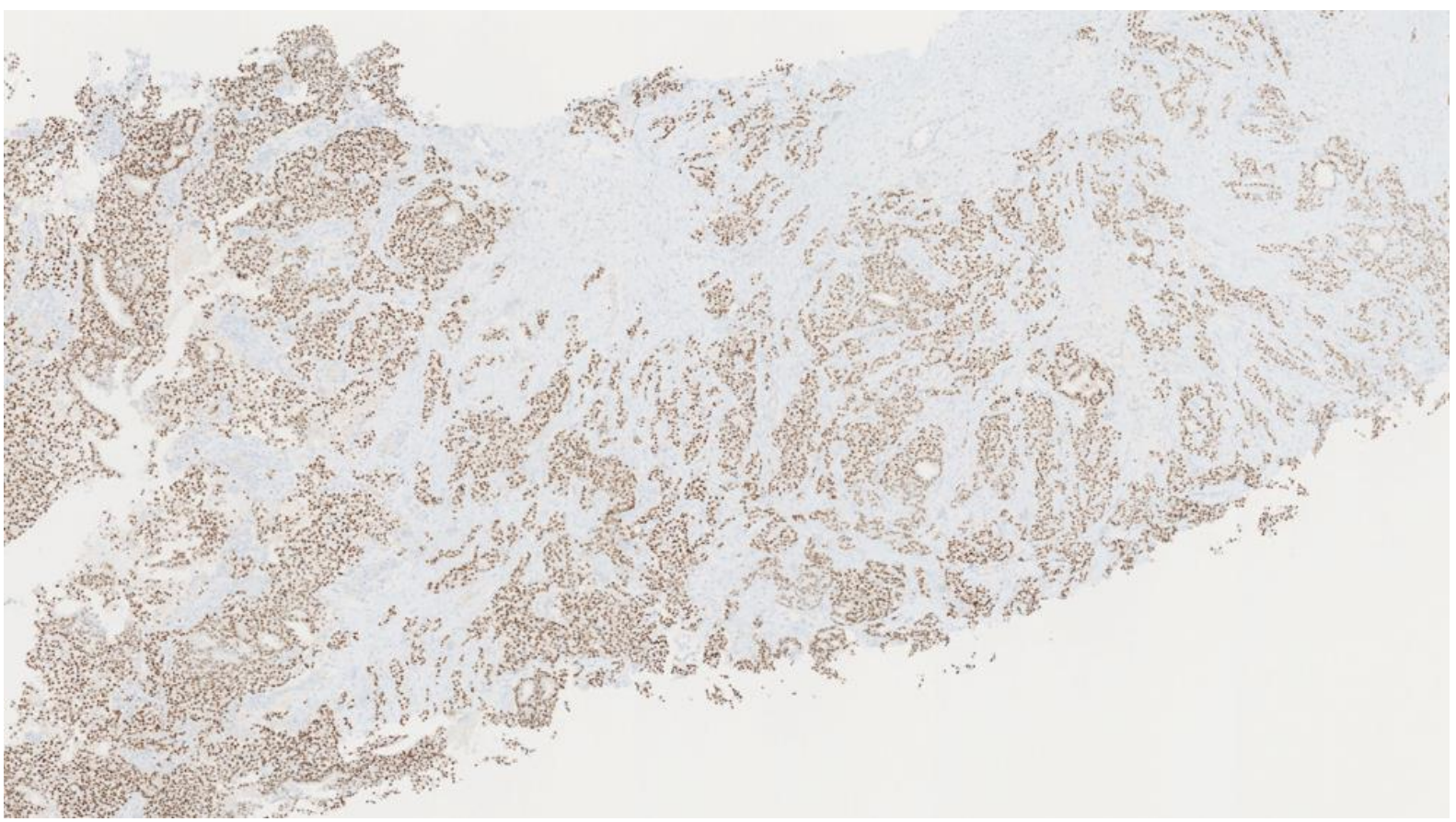

Figure 30: 47-year-old female with right breast metaplastic carcinoma.

Findings: There is strong nuclear expression in the tumor cells.

Technique: Microscopic examination. Medium-power (40x). Estrogen Receptor (ER) immunostaining from the core needle biopsy of a right breast complex solid and cystic mass. 


\begin{tabular}{|c|c|}
\hline Etiology & $\begin{array}{l}\text { - Typically arises when a solid mass gradually fills and dilates a lactiferous duct. } \\
\text { - Occasionally arises when a solid malignant mass secretes mucin/fluid or undergoes necrosis. }\end{array}$ \\
\hline Incidence & $\begin{array}{l}\text { - Complex solid and cystic masses found in approximately } 5 \% \text { of breast ultrasound examinations. } \\
\text { - Malignant potential of complex solid and cystic masses: between } 23 \text { and } 31 \% \text {. }\end{array}$ \\
\hline Gender Ratio & - Females > Males \\
\hline $\begin{array}{l}\text { Median Age } \\
\text { Predilection }\end{array}$ & $\begin{array}{l}\text { - Variable based on diagnosis: } \\
\text { ○ IDC: } 62 \\
\text { O DCIS: } 74.5 \\
\text { O Mucinous: } 62.3 \\
\text { O IPC: } 69.5\end{array}$ \\
\hline Risk Factors & $\begin{array}{l}\text { - Female gender } \\
\text { - Family history of breast cancer } \\
\text { - BRCA1/BRCA2 mutations } \\
\text { - Early age of menarche } \\
\text { - Late age of menopause } \\
\text { - Nulliparity } \\
\text { - Obesity } \\
\text { - Caucasian race }\end{array}$ \\
\hline Treatment & $\begin{array}{l}\text { - Surgical excision (mastectomy or breast conservation therapy) } \\
\text { - Adjuvant radiation, chemotherapy, and/or hormonal therapy }\end{array}$ \\
\hline Prognosis & $\begin{array}{l}\text { - Variable based on the receptor status of the tumor with triple negative carcinoma conferring the worst } \\
\text { prognosis and ER+/PR+/HER2- tumors conferring the best prognosis. }\end{array}$ \\
\hline Imaging Findings & $\begin{array}{l}\text { - Ultrasound: a cyst with thick walls }(>0.5 \mathrm{~mm}) \text {, thick septa }(>0.5 \mathrm{~mm}) \text {, an intra-cystic mass, or a cyst } \\
\text { demonstrating }>50 \% \text { solid components. }\end{array}$ \\
\hline
\end{tabular}

Table 1: Summary table for complex solid and cystic breast cancers

\begin{tabular}{|c|c|c|c|}
\hline & MAMMOGRAPHY & ULTRASOUND & MRI \\
\hline $\begin{array}{l}\text { Invasive } \\
\text { Ductal } \\
\text { Carcinoma } \\
\text { (IDC) }\end{array}$ & $\begin{array}{l}\text { - Irregularly shaped mass } \\
\text { - Spiculated margins } \\
\text { - Pleomorphic } \\
\text { microcalcifications in a } \\
\text { segmental distribution }\end{array}$ & $\begin{array}{l}\text { - Irregularly shaped mass } \\
\text { - Hypoechoic (occasionally complex mass with solid } \\
\text { and cystic components, secondary to necrosis) } \\
\text { - Not parallel orientation } \\
\text { - Angular or spiculated margins } \\
\text { - Posterior acoustic shadowing }\end{array}$ & $\begin{array}{l}\text { - Rim } \\
\text { enhancement } \\
\text { with centripetal } \\
\text { filling. }\end{array}$ \\
\hline $\begin{array}{l}\text { Ductal } \\
\text { Carcinoma } \\
\text { in-situ } \\
\text { (DCIS) }\end{array}$ & $\begin{array}{l}\text { - Microcalcifications } \\
\text { - Architectural distortion }\end{array}$ & $\begin{array}{l}\text { - Calcified DCIS: echogenic foci located within a } \\
\text { mass or a duct, associated with internal } \\
\text { microlobulations, or distributed in a branch pattern. } \\
\text { - Noncalcified DCIS: hypoechoic mass with } \\
\text { microlobulated margins and no posterior acoustic } \\
\text { features, or it may have a "pseudomicrocystic" } \\
\text { appearance. } \\
\text { - Less frequently, can present as an intracystic mass. }\end{array}$ & $\begin{array}{l}\text { - Non-mass } \\
\text { enhancement in } \\
\text { a linear or } \\
\text { segmental } \\
\text { distribution. } \\
\text { - Clustered ring } \\
\text { internal } \\
\text { enhancement } \\
\text { pattern. }\end{array}$ \\
\hline $\begin{array}{l}\text { Mucinous } \\
\text { Carcinoma }\end{array}$ & $\begin{array}{l}\text { - Microlobulated or } \\
\text { circumscribed margins }\end{array}$ & $\begin{array}{l}\text { - Complex mass with solid and cystic components. } \\
\text { - Increased vascularity of the solid components. } \\
\text { - Posterior acoustic enhancement. }\end{array}$ & - T2 hyperintense \\
\hline $\begin{array}{l}\text { Intracystic } \\
\text { Papillary } \\
\text { Carcinoma } \\
\text { (IPC) }\end{array}$ & $\begin{array}{l}\text { - Round or oval shaped mass } \\
\text { - Microlobulated or } \\
\text { circumscribed margins } \\
\text { - Often in the retroareolar region }\end{array}$ & - Complex mass with solid and cystic components. & $\begin{array}{l}\text { - Enhancement of } \\
\text { the cyst walls, } \\
\text { septa, and/or } \\
\text { mural nodules. }\end{array}$ \\
\hline
\end{tabular}

Table 2: Differential diagnosis table for complex solid and cystic breast cancers 


\section{ABBREVIATIONS}

BIRADS $=$ Breast imaging reporting and data system

$\mathrm{CT}=$ Computed tomography

DCIS = Ductal carcinoma in-situ

F18-FDG = 18F-2-fluoro-2-deoxyglucose

$\mathrm{H} \& \mathrm{E}=$ Hematoxylin and Eosin

HER-2 = Human epidermal growth factor receptor 2

IDC $=$ Invasive ductal carcinoma

IPC = Intracystic papillary carcinoma

MIP = Maximum intensity projection

NOS $=$ Not otherwise specified

$\mathrm{PET} / \mathrm{CT}=$ Positron emission tomography-computed tomography

SUV $=$ Standardized uptake value

SUVmax = Maximum standardized uptake value

\section{KEYWORDS}

Breast cancer; Ultrasound; BI-RADS; Complex cyst

\section{Online access}

This publication is online available at:

www.radiologycases.com/index.php/radiologycases/article/view/3712

\section{Peer discussion}

Discuss this manuscript in our protected discussion forum at: www.radiolopolis.com/forums/JRCR

\section{Interactivity}

This publication is available as an interactive article with scroll, window/level, magnify and more features.

Available online at www.RadiologyCases.com

Published by EduRad

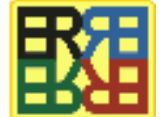

www.EduRad.org 